\title{
Study on the Single Scattering of Elastic Waves by a Cylindrical Fiber with a Partially Imperfect Bonding Using the Collocation Point Method
}

\author{
Jun Zhang $\mathbb{D}^{1,2}$ Longhai Zeng, ${ }^{1}$ Chuanlin $\mathrm{Hu} \mathbb{D}^{1},{ }^{3}$ and Wensheng Yan ${ }^{4}$ \\ ${ }^{1}$ College of Aerospace Engineering, Chongqing University, Chongqing 400044, China \\ ${ }^{2}$ Chongqing Key Laboratory of Heterogeneous Material Mechanics, Chongqing University, Chongqing 400044, China \\ ${ }^{3}$ State Key Laboratory of Silicate Materials for Architecture, Wuhan University of Technology, Wuhan 430070, China \\ ${ }^{4}$ Institute of Microstructure Technology (IMT), Karlsruhe Institute of Technology, Hermann-von-Helmholtz-Platz 1, \\ 76344 Eggenstein-Leopoldshafen, Germany
}

Correspondence should be addressed to Jun Zhang; mejzhang@cqu.edu.cn and Chuanlin Hu; chuanlin@whut.edu.cn

Received 9 January 2018; Revised 17 February 2018; Accepted 8 March 2018; Published 23 April 2018

Academic Editor: Nerio Tullini

Copyright (C) 2018 Jun Zhang et al. This is an open access article distributed under the Creative Commons Attribution License, which permits unrestricted use, distribution, and reproduction in any medium, provided the original work is properly cited.

\begin{abstract}
The single scattering of P- and SV-waves by a cylindrical fiber with a partially imperfect bonding to the surrounding matrix is investigated, which benefits the characterization of the behavior of elastic waves in composite materials. The imperfect interface is modelled by the spring model. To solve the corresponding single scattering problem, a collocation point (CP) method is introduced. Based on this method, influence of various aspects of the imperfect interface on the scattering of P-and SV-waves is studied. Results indicate that (i) the total scattering cross section (SCS) is almost symmetric about the axis $\alpha=\pi / 2$ with respect to the location ( $\alpha$ ) of the imperfect interface, (ii) imperfect interfaces located at $\alpha=0$ and $\alpha=\pi$ highly reduce the total SCS under a P-wave incidence and imperfect interfaces located at $\alpha=\pi / 2$ reduce the total SCS most significantly under SV-incidence, and (iii) under a P-wave incidence the SCS has a high sensitivity to the bonding level of imperfect interfaces when $\alpha$ is small, while it becomes more sensitive to the bonding level when $\alpha$ is larger under SV-wave incidence.
\end{abstract}

\section{Introduction}

A large number of interfaces exist in composite materials and play a very important role in the performance of composite materials, for example, the transmission of the load between the matrix and fibers. To simplify the analysis and calculation, the fibers/particulates are generally assumed to be perfectly bonded to the surrounding matrix [1-4]. However, this is not always the case, which has consequently led to the increased research effort to take the bonding conditions into account [5-16]. Among them, the debonding case [9-13, 1618], which is assumed to be a crack with noncontacting faces, has been well investigated. Specifically, Norris and Yang [1113] investigated the single scattering of antiplane shear and $\mathrm{P}$ - and SV-waves by a partially debonded fiber through the expansion of the crack opening displacement (COD) or the stress at the neck in terms of Chebyshev polynomials. The results indicated that a resonance happens at low frequencies when the neck joining the fiber to the matrix is thin. Recently, Fang et al. [16] extended this method to the case of pipelines. Based on the same method, Kim [9] studied the effects of interface debonding on the attenuation and speed of antiplane shear wave propagating in fiber-reinforced composites. The corresponding static effective mechanical properties of fiber-reinforced composites were obtained from the asymptotic solutions of dynamic harmonic analysis by Liu and Kriz [10]. Besides the technique proposed by Norris and Yang [11-13], Y. S. Wang and D. Wang [17, 18] proposed a different method, where the dislocation density function was introduced and the interface conditions were transformed to standard Cauchy singular integral equations. Solving these equations gives rise to the COD at each debonding/crack. Compared with the method of Norris and Yang, the one proposed by Y. S. Wang and D. Wang has a high convergence 
rate especially when the size of cracks is small. Besides these theoretical investigations, numerical simulations using the boundary element methods were also conducted, for example, Biwa and Shibata $[19,20]$ and Sato and Shindo [21].

The perfect bonding and the debonding are the two extreme cases of the bonding conditions of interfaces. Actually, there are other conditions which extensively exist between the perfect bonding and the debonding, that is, imperfect condition. For this reason, several theoretical models were proposed during the past few years [22-24]. Among them, the spring model [22] attracted the most attention. In the spring model, the stresses are assumed to be continuous across the interface, while the displacements jump. Furthermore, the displacement jump is assumed to be linearly proportional to the stress at the interface. The stress to the displacement jump ratio is defined as the stiffness of springs. Thus, the stiffness of the spring reflects the bonding level of the imperfect interface. It is clear that when the stiffness of the spring tends towards infinity, the imperfect bonding becomes the usual perfect bonding, while it approaches the debonding when the stiffness approaches zero.

Using the above-introduced spring model, the effective mechanical properties of composite materials, such as the effective modulus $[25,26]$, thermal expansion coefficient [27], phase velocity, and attenuation [5, 8, 28], have been extensively investigated by taking the imperfect bonding into account. The spring model has also been used to study the influence of the imperfect bonding effect on the behaviors of the underground tunnels under various dynamic loadings $[15,24,29,30]$.

It is worthwhile to mention that, in most of the previous studies, the whole interface between each fiber/particulate and the matrix was usually assumed to simultaneously enter the imperfect bonding condition. However, this is not real case, assuming that the interface between each fiber and the surrounding matrix deteriorates as a process would be more reasonable. In this regard, less attention has been paid except for the partial debonding case. The limited works include those of Lopez-Realpozo et al. [31] and Guinovart-Díaz et al. [32], where the effect of the partially imperfect bonding on the effective mechanical properties of fiber-reinforced composites was investigated. To the best of our knowledge, investigation on the influence of this partially imperfect bonding of interfaces on the behavior of P- and SV-waves in composites is still scarce. It is well known that the study of the single scattering is crucial to understanding the overall behavior of elastic waves in composites. Towards this end, in this work, the single scattering of P- and SV-waves by a cylindrical fiber with a partially imperfect bonding to the surrounding matrix is studied. Compared with the single scattering with a fully perfect or imperfect interface, solving the single scattering with a partially imperfect bonding is much more complicated, since the conditions at the interface become discontinuous. In this work, the imperfect bonding is characterized by the spring model and a CP method is introduced to solve the corresponding single scattering problem.

In the following sections, firstly, the single scattering problem is formulated. Then, the CP method is introduced to solve the single scattering problem. After that, the influence of various aspects of the partially imperfect bonding on the single scattering is extensively studied numerically. Finally, a short conclusion is drawn.

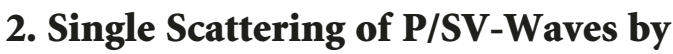 a Fiber with a Partially Imperfect Bonding}

2.1. Governing Equations for P-and SV-Waves in $2 D$ Problems. The governing equations for $\mathrm{P}$ - and $\mathrm{SV}$-waves in $2 \mathrm{D}$ problems with a homogeneous medium are decoupled based on the Helmholtz decomposition. In harmonic analysis, the two equations for $\mathrm{P}$ - and $\mathrm{SV}$-waves can be expressed as

$$
\begin{aligned}
& \left(\nabla^{2}+k_{l}^{2}\right) \phi=0 \\
& \left(\nabla^{2}+k_{s}^{2}\right) \varphi=0,
\end{aligned}
$$

where $\phi$ and $\varphi$ denote the displacement potentials for P- and $S V$-waves, respectively; and $\nabla^{2}$ is the Laplace operator. The symbols $k_{l}$ and $k_{s}$ are the wavenumbers of the P- and SVwaves, and the subscripts $l$ and $s$ represent the longitudinal and transverse wave. The wavenumbers are related to material properties as

$$
\begin{aligned}
& k_{l}=\frac{\omega}{\sqrt{(\lambda+2 \mu) / \rho}} ; \\
& k_{s}=\frac{\omega}{\sqrt{\mu / \rho}},
\end{aligned}
$$

where $\omega$ is the circular frequency, $\lambda$ is the Lame constant, $\mu$ is the shear modulus, and $\rho$ is the mass density. In the polar coordinate system, the displacements and stresses are expressed as

$$
\begin{aligned}
u_{r}= & \frac{\partial \phi}{\partial r}+\frac{1}{r} \frac{\partial \varphi}{\partial \theta} \\
u_{\theta}= & \frac{1}{r} \frac{\partial \phi}{\partial \theta}-\frac{\partial \varphi}{\partial r} \\
\sigma_{r r}= & (\lambda+2 \mu) \frac{\partial^{2} \phi}{\partial r^{2}}+\lambda\left(\frac{1}{r^{2}} \frac{\partial^{2} \phi}{\partial \theta^{2}}+\frac{1}{r} \frac{\partial \phi}{\partial r}\right) \\
& +2 \mu\left(\frac{1}{r} \frac{\partial^{2} \varphi}{\partial r \partial \theta}-\frac{1}{r^{2}} \frac{\partial \varphi}{\partial \theta}\right) \\
\sigma_{r \theta}= & \mu\left(-\frac{2}{r^{2}} \frac{\partial \phi}{\partial \theta}+\frac{2}{r} \frac{\partial^{2} \phi}{\partial r \partial \theta}+\frac{1}{r^{2}} \frac{\partial^{2} \varphi}{\partial \theta^{2}}-\frac{\partial^{2} \varphi}{\partial r^{2}}+\frac{1}{r} \frac{\partial \varphi}{\partial r}\right)
\end{aligned}
$$

where $u_{r}$ and $u_{\theta}$ represent the radial and circumferential displacements, and $\sigma_{r r}$ and $\sigma_{r \theta}$ are the radial and tangential stresses. The formula in ((1)-(3)) can be found in numerous classic books, of which we mention the monograph by Mow and Pao [33]. They are copied here to maintain the integrity of this work.

2.2. Problem Formulation of the $2 D$ Singe Scattering of $P$ - and $S V$-Waves. Figure 1 shows a schematic of the single scattering 


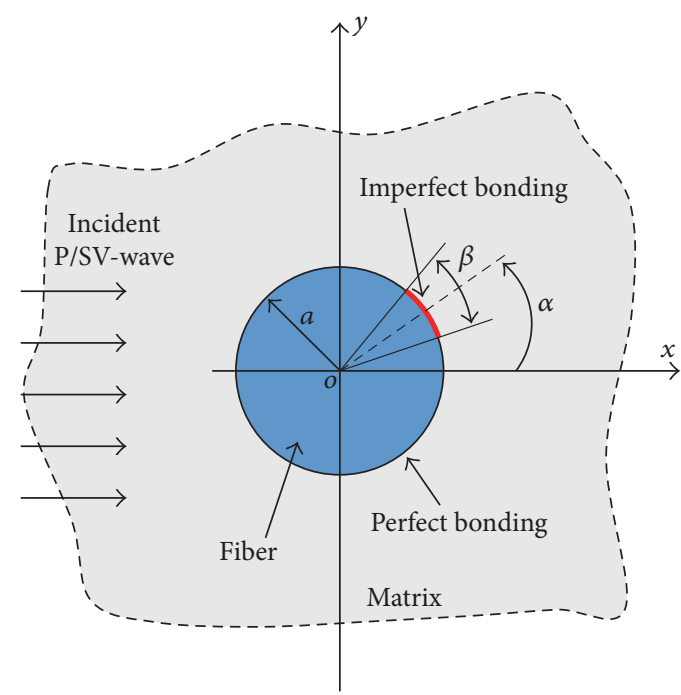

FIGURE 1: Scattering of a plane P- or SV-wave by a cylindrical fiber with a partially imperfect bonding to the matrix. The range of the imperfect bonding is denoted by the red solid arc.

of a plane P- or SV-wave by a cylindrical fiber partially imperfectly bonded to the surrounding matrix. The radius of the fiber is $a$. The range of the imperfect interface is represented by angle $\beta$, as indicated by the red solid curve shown in Figure 1 . The symmetric axis of the imperfect interface is at angle $\alpha$ with the horizontal axis. It is clear that when $\beta=0$, the whole fiber is fully perfectly bonded to the matrix, and the whole interface becomes a fully imperfect bonding as $\beta=2 \pi$. In this work, the imperfect bonding is modelled using the spring model introduced before, where it is characterized using two springs distributed along the radial and circumferential directions, with stiffness $K_{n}$ and stiffness $K_{t}$, respectively. The incident wave is a plane P- or SV-wave propagating in the horizontal direction. In the polar coordinate system, general solutions to (1) are the products of Bessel functions and trigonometric functions. Hence, for harmonic analysis, the incident wave, the wave scattered by the fiber, and the wave fields inside the fiber can be expressed as series of cylindrical wave functions with the omission of $e^{-\mathbf{i} \omega t}$ :

$$
\begin{aligned}
\phi^{i n c}(r, \theta) & =e^{\mathrm{i} k_{l}^{m} x}=\sum_{n=-\infty}^{\infty} \mathbf{i}^{n} J_{n}\left(k_{l}^{m} r\right) e^{\mathrm{i} n \theta} \\
\phi^{m}(r, \theta) & =\sum_{n=-\infty}^{\infty} A_{n} H_{n}^{(1)}\left(k_{l}^{m} r\right) e^{\mathrm{i} n \theta} \\
\varphi^{m}(r, \theta) & =\sum_{n=-\infty}^{\infty} B_{n} H_{n}^{(1)}\left(k_{s}^{m} r\right) e^{\mathrm{i} n \theta} \\
\phi^{f}(r, \theta) & =\sum_{n=-\infty}^{\infty} C_{n} J_{n}\left(k_{l}^{f} r\right) e^{\mathrm{i} n \theta} \\
\varphi^{f}(r, \theta) & =\sum_{n=-\infty}^{\infty} D_{n} J_{n}\left(k_{s}^{f} r\right) e^{\mathrm{i} n \theta},
\end{aligned}
$$

where $(r, \theta)$ are the polar coordinates with origins at the center of the fiber. The wavenumbers $k_{l, s}^{\alpha}=\omega / c_{l, s}^{\alpha}(\alpha=$ $m$ and $f$ ), where $c_{l, s}^{\alpha}$ is the wave speed of longitudinal (l) wave or transverse $(s)$ wave. The superscripts $m$ and $f$ indicate quantities associated with the matrix and the fiber, respectively. $J_{n}(\cdot)$ and $H_{n}^{(1)}(\cdot)$ are the Bessel and Hankel functions of the first kind; $\mathbf{i}=\sqrt{-1}$ is the imaginary unit; and $A_{n}, B_{n}, C_{n}$, and $D_{n}$ are the unknown coefficients, which are to be determined by the conditions at the interface. In the current case, the conditions of stresses at the interface are listed as

$$
\begin{aligned}
& \left.\sigma_{r r}^{m}\right|_{r=a}=\left.\sigma_{r r}^{f}\right|_{r=a}, \\
& \left.\sigma_{r \theta}^{m}\right|_{r=a}=\left.\sigma_{r \theta}^{f}\right|_{r=a}, \\
& \quad \theta \in[0,2 \pi] .
\end{aligned}
$$

And the conditions for the radial and circumferential displacements at the interface can be expressed as

$$
\begin{aligned}
& K_{n}\left[\left.u_{r}^{m}\right|_{r=a}-\left.u_{r}^{f}\right|_{r=a}\right]= \begin{cases}\left.\sigma_{r r}^{f}\right|_{r=a}, & \theta \in \beta \\
0, & \text { otherwise }\end{cases} \\
& K_{t}\left[\left.u_{\theta}^{m}\right|_{r=a}-\left.u_{\theta}^{f}\right|_{r=a}\right]= \begin{cases}\left.\sigma_{r \theta}^{f}\right|_{r=a}, & \theta \in \beta \\
0, & \text { otherwise. }\end{cases}
\end{aligned}
$$

The total displacement potential in the matrix is the summation of the incident wave and the scattered wave, while the quantities in the fiber are only of the refracted one. By substituting the total displacement potentials in the matrix and the fiber expressed in (4) into (3), the displacements and stresses in the matrix and the fiber are obtained. Then, the interface conditions in (5) and (6) can be expressed in 
terms of the expansion coefficients $A_{n}, B_{n}, C_{n}$, and $D_{n}$. Firstly, based on (5), two equations are established for the expansion coefficients as

$$
\left[\begin{array}{llll}
M_{31} & M_{32} & M_{33} & M_{34} \\
M_{41} & M_{42} & M_{43} & M_{44}
\end{array}\right]\left[\begin{array}{c}
A_{n} \\
B_{n} \\
C_{n} \\
D_{n}
\end{array}\right]=\left[\begin{array}{l}
b_{3} \\
b_{4}
\end{array}\right]
$$

$$
n=-\infty, \infty \text {. }
$$

The detailed expressions for $M_{i j}$ and $b_{i}(i=3,4$ and $j=$ $1,2,3,4)$ are listed in Appendix A. After a simple mathematical operation on (7), the coefficients $A_{n}$ and $B_{n}$ can be expressed in terms of $C_{n}$ and $D_{n}$ as

$$
\left[\begin{array}{l}
A_{n} \\
B_{n}
\end{array}\right]=\left[\begin{array}{l}
\beta_{1}(n) \\
\beta_{2}(n)
\end{array}\right]+\left[\begin{array}{ll}
\alpha_{11}(n) & \alpha_{12}(n) \\
\alpha_{21}(n) & \alpha_{22}(n)
\end{array}\right]\left[\begin{array}{l}
C_{n} \\
D_{n}
\end{array}\right]
$$

with $\left[\begin{array}{l}\beta_{1}(n) \\ \beta_{2}(n)\end{array}\right]=\left[\begin{array}{ll}M_{31} & M_{32} \\ M_{41} & M_{42}\end{array}\right]^{-1}\left[\begin{array}{l}b_{3} \\ b_{4}\end{array}\right]$ and $\left[\begin{array}{ll}\alpha_{11}(n) & \alpha_{12}(n) \\ \alpha_{21}(n) & \alpha_{22}(n)\end{array}\right]=$ $-\left[\begin{array}{ll}M_{31} & M_{32} \\ M_{41} & M_{42}\end{array}\right]^{-1}\left[\begin{array}{ll}M_{33} & M_{43} \\ M_{43} & M_{44}\end{array}\right]$, where the superscript “- 1 ” means the inverse operation on a square matrix. Then, the remaining conditions for the radial and circumferential displacements in (6) can be expressed in terms of coefficients $C_{n}$ and $D_{n}$ :

$$
\begin{array}{r}
\sum_{n=-\infty}^{\infty} c_{j 1}(n, \theta) C_{n}+\sum_{n=-\infty}^{\infty} c_{j 2}(n, \theta) D_{n}=\sum_{n=-\infty}^{\infty} h_{j}(n, \theta) \\
j=1,2,3,4 .
\end{array}
$$

The details for $c_{i j}$ and $h_{i}(i=1,2,3,4 ; j=1,2)$ are referred to in Appendix B. Note that, in (9), $\theta$ is within the imperfectly bonded interface as $j=1$ and 3 and is within the perfectly bonded interface for $j=2$ and 4 . Since $\theta$ does not vary from 0 to $2 \pi$ in any equation of (9), the orthogonality for the sine and cosine functions is not available. Therefore, analytical solutions for $C_{n}$ and $D_{n}$ are difficult to be obtained.

\section{Scattering Cross Section and the Far-Field Scattering Magnitude}

In order to characterize the overall behavior of coherent waves in composites with randomly distributed fibers/particulates, over the past several decades, extensive theoretical models have been proposed including the $T$-matrix method [34-36], the self-consistent method [37-40], and the phenomenological model (also known as the independent scattering model) $[1,2,41,42]$. It is noted that all these theoretical models necessitate the solution of the corresponding single scattering problem to obtain the parameters, such as the total SCS and the far-field scattering magnitude. Therefore, the current study is indispensable, especially taking the imperfection of interfaces into account. In the current case (2D P- and SV-waves), the total SCS can be calculated by

$$
\sigma_{s}(\alpha, \beta)=\frac{4}{k_{l}^{m}} \sum_{n=-\infty}^{\infty}\left(\left|A_{n}\right|^{2}+\left|B_{n}\right|^{2}\right) .
$$

The far-field scattering magnitude $(r \rightarrow \infty)$ is defined as

$$
\begin{aligned}
\vec{u}^{s}= & u_{r}^{s} \vec{e}_{r}+u_{\theta}^{s} \vec{e}_{\theta} \\
= & \mathbf{i} k_{l}^{m} \sqrt{\frac{2}{\pi k_{l}^{m} r} e^{\mathbf{i}\left(k_{l}^{m} r-\pi / 4\right)} f_{l}(\theta) \vec{e}_{r}} \\
& +\mathbf{i} k_{s}^{m} \sqrt{\frac{2}{\pi k_{s}^{m} r}} e^{\mathbf{i}\left(k_{s}^{m} r-\pi / 4\right)} f_{s}(\theta) \vec{e}_{\theta} .
\end{aligned}
$$

Here a variable with an arrow represents a vector. The superscript $s$ denotes quantities of the scattered wave. By substituting the expression of the scattered wave into the above equation, the far-field scattering magnitudes for the scattered P- and SV-waves can be expressed as [13]

$$
\begin{aligned}
& f_{l}(\theta)=\sum_{n=-\infty}^{\infty}(-\mathbf{i})^{n} A_{n} e^{\mathbf{i} n \theta} \\
& f_{s}(\theta)=\sum_{n=-\infty}^{\infty}(-\mathbf{i})^{n} B_{n} e^{\mathbf{i} n \theta} .
\end{aligned}
$$

$\sigma_{s}(\theta), f_{l}(\theta)$, and $f_{s}(\theta)$ are evaluators of the scattering capability of the fiber. Accurate calculation of these parameters plays a critical role in these theoretical models.

\section{The Collocation Point Method}

In this study, the CP method is adopted to solve (9). First, the infinite series in (4) are truncated with the maximum order $n_{\max }$. Then, the number of the unknowns $C_{n}$ and $D_{n}$ in $(9)$ becomes $\left(2 n_{\max }+1\right)$. In order to solve these unknowns, both the first $(j=1,2)$ and second $(j=3,4)$ group equations in (9) are forced to be satisfied at $\left(2 n_{\max }+1\right)$ CPs. In this work, these CPs are distributed symmetrically about the partially imperfectly and perfectly bonded interfaces, as shown in Figure 2. Additionally, these CPs are required to be distributed as uniformly as possible on the whole interface. Then, a linear system of size $2 \times\left(2 n_{\max }+1\right)$ is established for $C_{n}$ and $D_{n}$. Solving this linear system yields $C_{n}$ and $D_{n}$ ( $\left.n=-n_{\max }, n_{\max }\right)$; the coefficients $A_{n}$ and $B_{n}$ can then be calculated according to (8). Then the displacements and stresses at each point inside the fiber and the matrix can be calculated.

To check the convergence of this CP method, the total SCS by a partially debonded fiber $(\alpha=0$ and $\beta=2 \pi / 3)$ under different $n_{\max }$ is calculated and plotted in Figure 3. In this case, the materials for the fiber and the matrix are glass and epoxy, whose detailed properties are listed in Table 1 . The results show that the calculated SCS converges to a constant as $n_{\max }$ increases. Based on this convergence analysis, in this study, $n_{\max }$ is set as 10 .

Next, three examples are calculated to validate the application of the CP method. Figure 4 shows the calculated SCS by a partially imperfectly bonded fiber changing with the stiffness at $k_{l}^{m} a=3.0$. The materials used are the same as those in Figure 3. The dash lines represent values of the debonding and the perfect bonding cases obtained in [13]. 


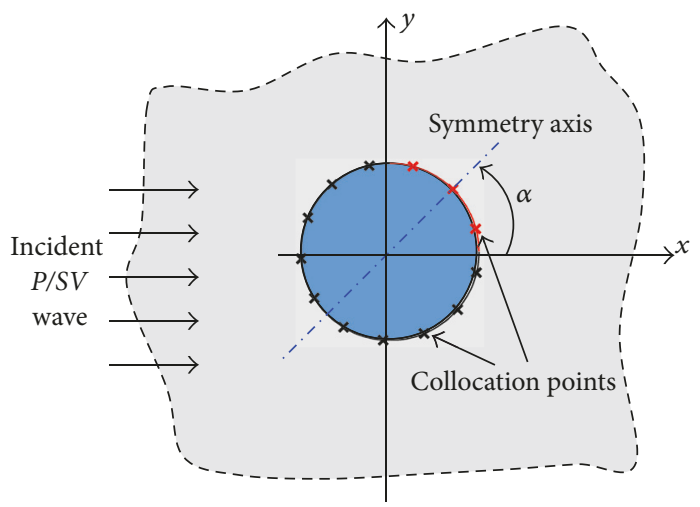

Figure 2: Distribution of CPs denoted by the " $x$ " symbol. At least one CP should be distributed on the imperfectly bonded interface as long as $\beta$ is not equal to 0 . In this figure, $\alpha=\pi / 4, \beta=\pi / 2$, and $n_{\max }=6$.

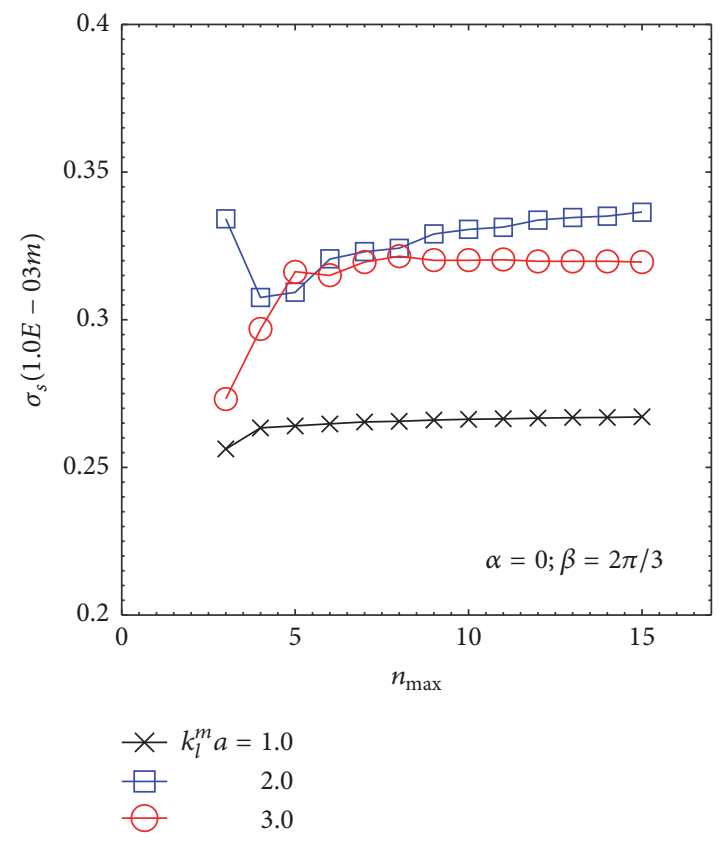

Figure 3: Convergence study of the CP method using the debonding case considered in [13]. In the CP method, the stiffness $K_{n} / K_{n}^{*}=$ $K_{t} / K_{t}^{*}=10^{-6}$ represents the debonding. In this work, $K_{n}^{*}$ and $K_{t}^{*}$ are defined as $K_{n}^{*}=0.5\left(\lambda^{m}+\lambda^{f}\right) / 0.1 a$ and $K_{t}^{*}=0.5\left(\mu^{m}+\mu^{f}\right) / 0.1 a$.

TABLE 1: Materials used in the current work $[4,13]$.

\begin{tabular}{lccc}
\hline Material & $\lambda(\mathrm{GPa})$ & $\mu(\mathrm{GPa})$ & $\rho\left(\mathrm{kg} / \mathrm{m}^{3}\right)$ \\
\hline $\mathrm{SiC}$ & 92.1 & 177.0 & 3200 \\
Ti-alloy & 103.0 & 44.8 & 5400 \\
Glass & 0.7367 & 1.43 & 2550 \\
Epoxy & 0.8895 & 1.28 & 1250 \\
\hline
\end{tabular}

The results from this study approach the exact solutions when the stiffness tends to zero and infinity. Moreover, the calculated results under different $\beta$ tend to the same value as the stiffness increases, which follows the fact that all the imperfect bonding of different widths becomes the perfect one as the stiffness is large enough. In the following two calculations, the matrix is Ti-alloy and the fiber is made up of
$\mathrm{SiC}$. The detailed properties of the two materials are tabulated in Table 1. Figure 5 shows the calculated far-field scattering magnitude patterns for the scattered $\mathrm{P}$ - and SV-waves under a plane $\mathrm{P}$-wave incidence with a normalized frequency $k_{l}^{m} a=$ 1.0. The imperfect bonding is located at $\alpha=0$ with a width $\beta=\pi / 2$. As shown in Figure 5, patterns at several different bonding levels are plotted and compared with that of the fully perfect bonding case. Results show that the pattern with a partially imperfect bonding approaches that of the fully perfect bonding case as the stiffness $K_{n}$ and stiffness $K_{t}$ increase, which agrees with the theoretical expectation.

Figure 6 shows the calculated far-field scattering magnitude patterns of the scattered P- and SV-waves by a fiber with a fully $(\beta=2 \pi)$ imperfect bonding under a P-wave incidence at a normalized frequency $k_{l}^{m} a=3.0$. Results show that patterns of both the scattered P- and SV-waves approach those 


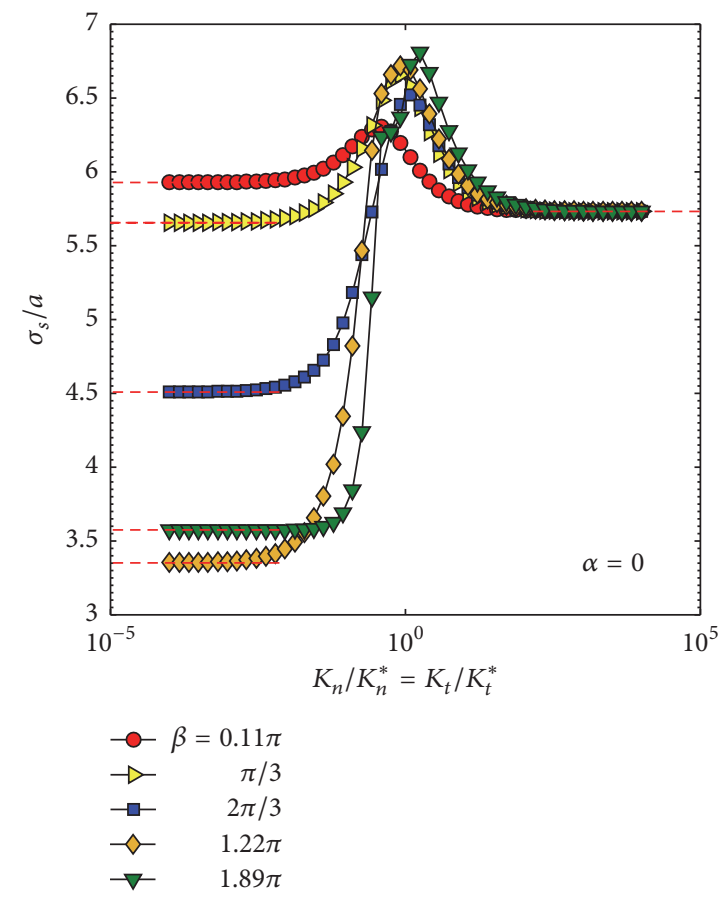

FIGURE 4: The calculated SCS by a partially imperfectly bonded fiber as a function of the stiffness in the range of $10^{-4}$ to $10^{4}$.

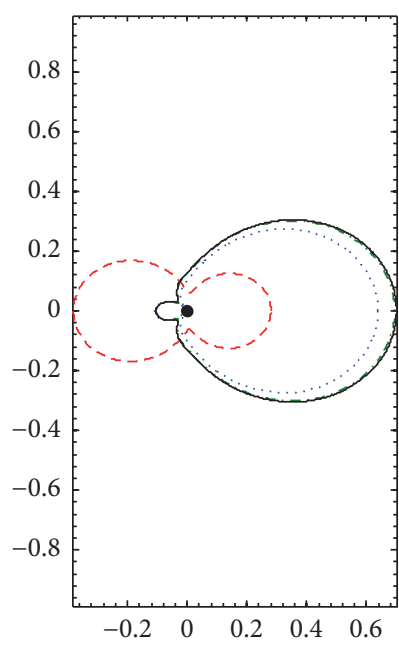

(a)

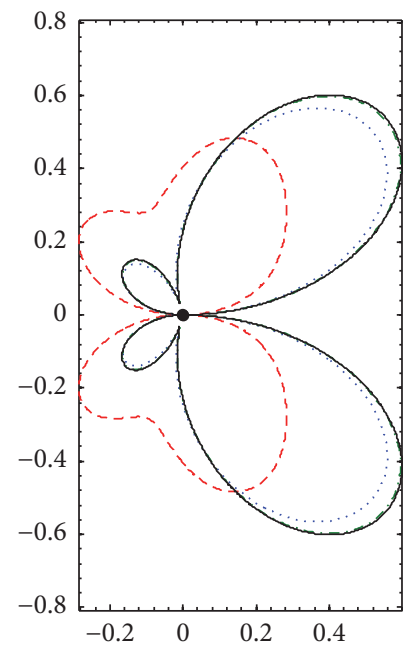

(b)

Figure 5: The far-field scattering magnitude patterns of the scattered (a) P- and (b) SV-waves by a fiber with a partially imperfect bonding to the matrix, under a plane P-wave incidence (dashed line: $K_{n} / K_{n}^{*}=K_{t} / K_{t}^{*}=10^{-1}$; dotted line: $K_{n} / K_{n}^{*}=K_{t} / K_{t}^{*}=10^{0}$; dash dotted line: $K_{n} / K_{n}^{*}=K_{t} / K_{t}^{*}=10^{1}$; solid line: perfect bonding).

of the cavity one as the stiffness $K_{n}$ and stiffness $K_{t}$ decrease, which is also in agreement with the theoretical expectation.

Figures 4, 5, and 6 indicate that the CP method performs well to calculate the total SCS and the far-field scattering magnitude for the single scattering problem of $\mathrm{P}$ - or SV-waves by a fiber with a partially imperfect bonding to the matrix. But, it should be reminded that the current $\mathrm{CP}$ method is not a proper technique to calculate the corresponding crack opening displacements and the stresses at the interface, especially around the tips of the imperfect interface. Moreover, the results show that the spring model with the 


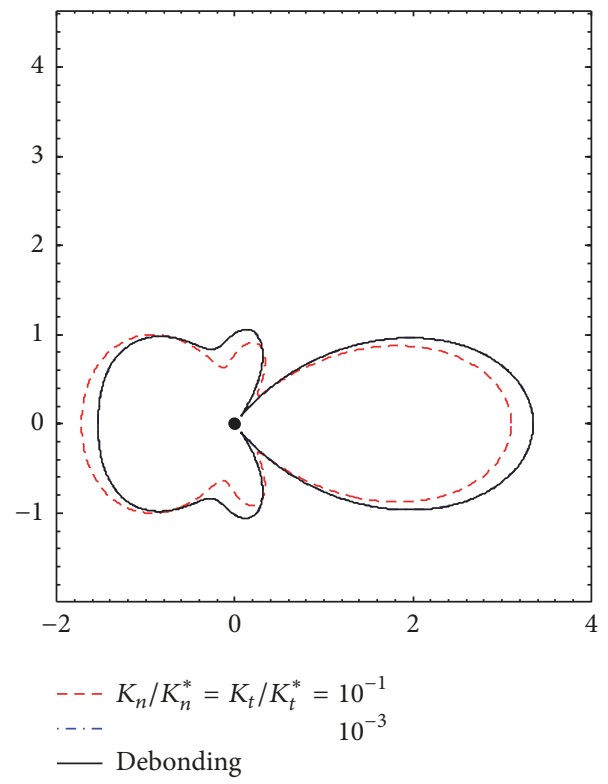

(a)

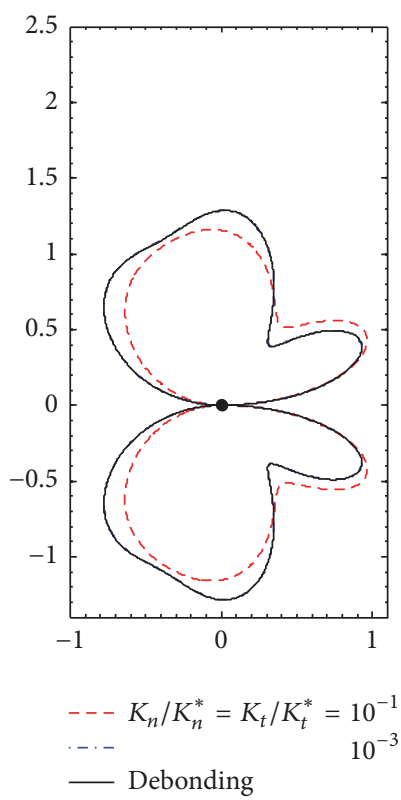

(b)

FIGURE 6: Comparison of the calculated far-field scattering patterns of the scattered (a) P-wave and (b) SV-wave by a fiber with a fully imperfect bonding to the matrix under a P-wave incidence versus those of the debonding case (cavity).

setting of $K_{n} / K_{n}^{*}=K_{t} / K_{t}^{*}=10^{1}$ and $K_{n} / K_{n}^{*}=K_{t} / K_{t}^{*}=$ $10^{-3}$ properly modelled the perfect bonding and debonding situations, respectively.

\section{Effects of the Partially Imperfect Bonding on the Single Scattering}

5.1. Effects of $\alpha$ and $\beta$. In this section, the influence of $\alpha$ and $\beta$ on the single scattering of $\mathrm{P}$ - and SV-waves is investigated. The stiffness of the imperfectly bonded interface is set as $K_{n} / K_{n}^{*}=K_{t} / K_{t}^{*}=10^{-2}$, which represents a serious imperfection close to the debonding. Figure 7 shows the relative divergence of the calculated $\sigma_{s}$ under different $\alpha$ and $\beta$ from that of the fully perfect bonding, under a $\mathrm{P}$-wave incidence. Based on the results shown in this figure, several conclusions are obtained. First, it is clear that $\sigma_{s}$ is almost symmetric about the axis $\alpha=\pi / 2$. Second, within the range of investigated $\beta$, the partially imperfect bonding located at $\alpha=0$ and $\pi$ reduces $\sigma_{s}$ the most. In contrast, the partially imperfect bonding located at $\alpha=\pi / 2$ has almost no effect on $\sigma_{s}$ compared with the fully perfect bonding, especially as the frequency is relatively high; all figures except Figure 7(a) show this. Theoretically, this fact benefits the identification of the specific location of the imperfection. Finally, as the frequency increases to values larger than $k_{l}^{m} a=2.0$, the partially imperfect bonding always reduces $\sigma_{s}$ compared with the fully perfect bonding, wherever the partially imperfect bonding is located.

The corresponding results under the SV-wave incidence are plotted in Figure 8. The frequencies of the incident wave are the same for each pair of figures in Figures 7 and 8. Similar to the results of the P-wave incidence, $\sigma_{s}$ under the
SV-wave incidence is also symmetric about the axis $\alpha=\pi / 2$. Moreover, for each specific $\beta$, the partially imperfect bonding located at $\alpha=\pi / 2$ reduces $\sigma_{s}$ the most. Similarly, this fact is also beneficial to the identification of the location of the imperfection. In addition, contrary to the P-wave incidence, the partially imperfect bonding always reduces $\sigma_{s}$ at relatively lower frequencies rather than at higher frequencies.

5.2. The Stiffness Effect. In this section, influence of stiffness $K_{n}$ and stiffness $K_{t}$ of the imperfect interface on the single scattering is studied. Figure 9 shows the calculated $\sigma_{s}$ changing with $K_{n}$ and $K_{t}$ at three different frequencies under the Pand SV-wave incidence, respectively. The partially imperfect bonding is located at $\alpha=0$ with a width $\beta=\pi / 4$. It is clear that $\sigma_{s}$ is sensitive to $K_{n}$ and insensitive to $K_{t}$ under the P-wave incidence. On the contrary, under the SV-wave incidence, $\sigma_{s}$ becomes sensitive to $K_{t}$ rather than $K_{n}$.

Definitely, the sensitivity of $\sigma_{s}$ to $K_{n}$ and $K_{t}$ is affected by the location of the partially imperfect bonding, based on the results shown in Figures 7 and 8. In this work, this sensitivity is defined using the slope $\kappa$ of the solid line shown in Figure 10, which is the linear fitting to the curve within $\left(10^{-1}, 10^{0}\right)$. A larger absolute value of $\kappa$ means $\sigma_{s}$ has a higher sensitivity to the stiffness of the imperfectly bonded interface. Figure 11 shows values of $|\kappa|$ under the P-wave incidence as the imperfect bonding is located at different positions. In this figure, $\beta=\pi / 4$. The results indicate that $\sigma_{s}$ is more sensitive to $K_{n}$ than to $K_{t}$. Moreover, $\sigma_{s}$ changes significantly in response to $K_{n}$ when $\alpha$ is small, as Figure 11(a) shows. In addition, the sensitivity of $\sigma_{s}$ to $K_{t}$ reaches a maximum as $\alpha$ equals approximately $\pi / 4$. Figure 11 (c) shows the sensitivity of $\sigma_{s}$ to both $K_{n}$ and $K_{t}$. 


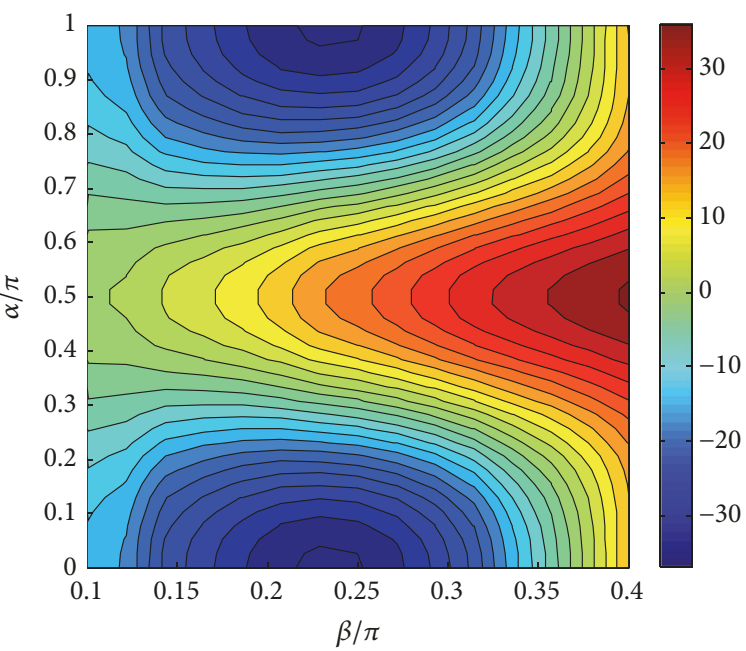

(a) $k_{l}^{m} a=1.0$

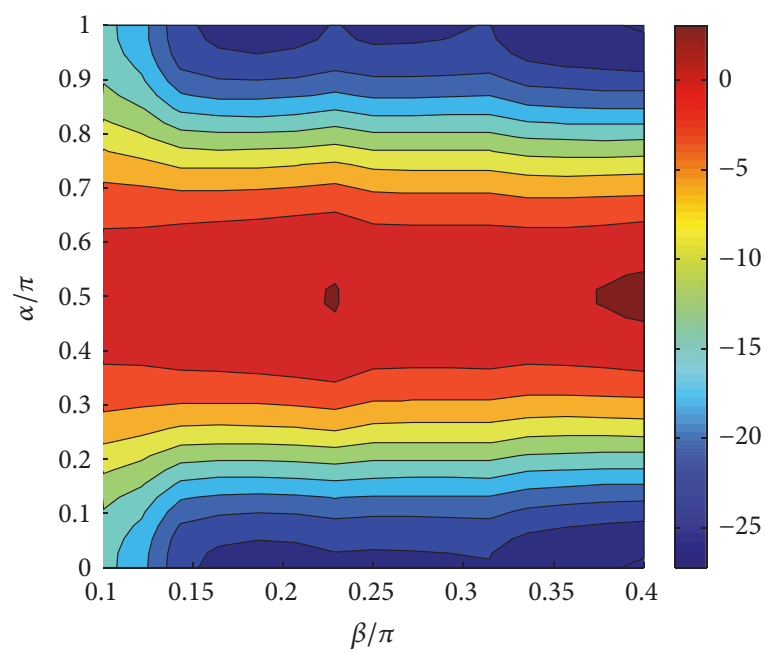

(c) $k_{l}^{m} a=2.0$

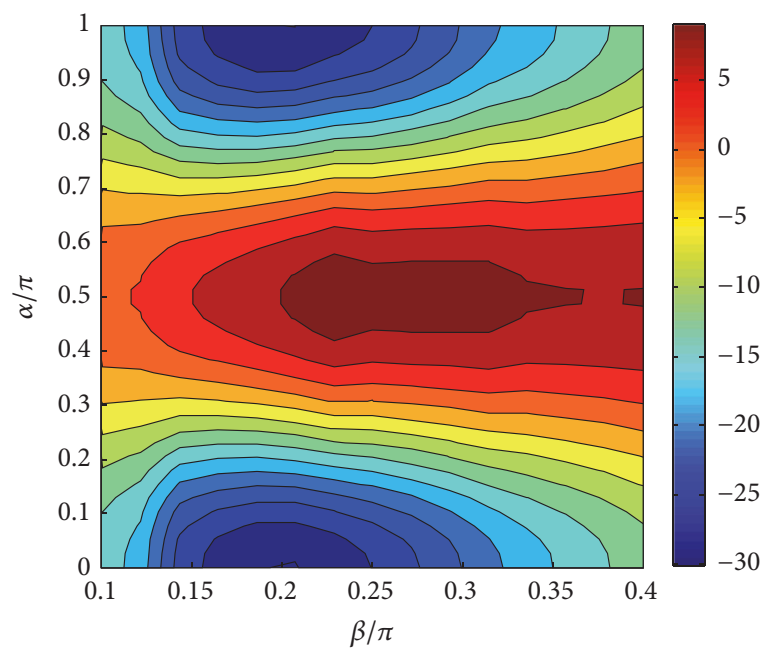

(b) $k_{l}^{m} a=1.5$

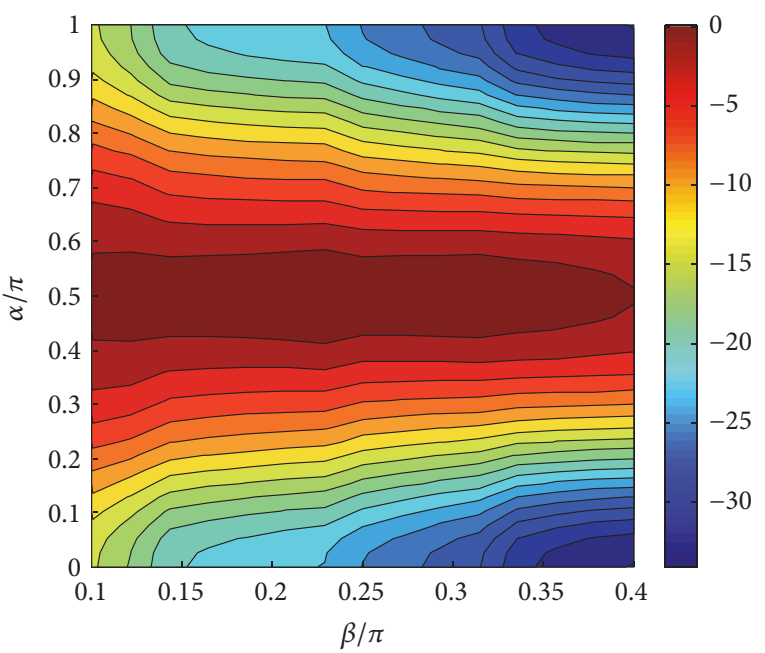

(d) $k_{l}^{m} a=2.5$

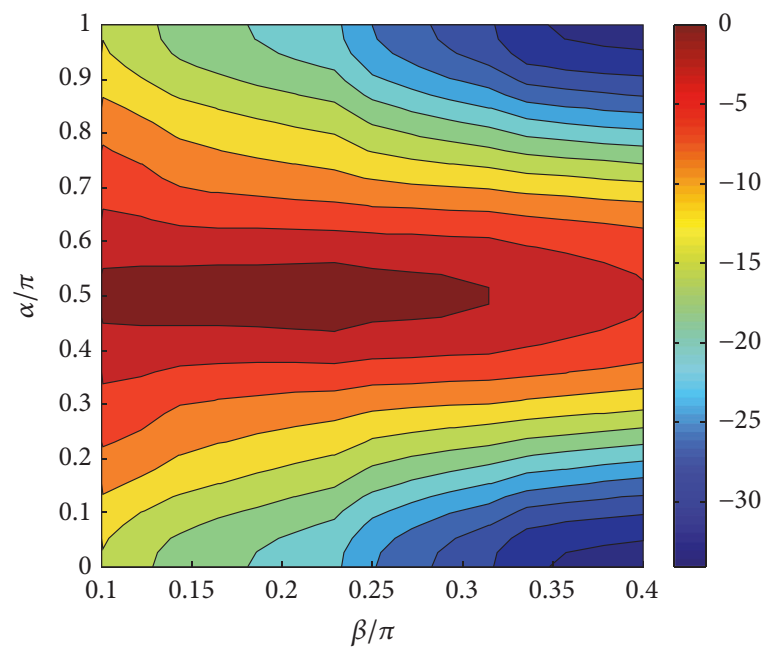

(e) $k_{l}^{m} a=3.0$

FIGURE 7: Relative difference (\%) of the calculated $\sigma_{s}$ by a fiber with a partially imperfect bonding and with the fully perfect bonding, under the P-wave incidence at several different normalized frequencies. 


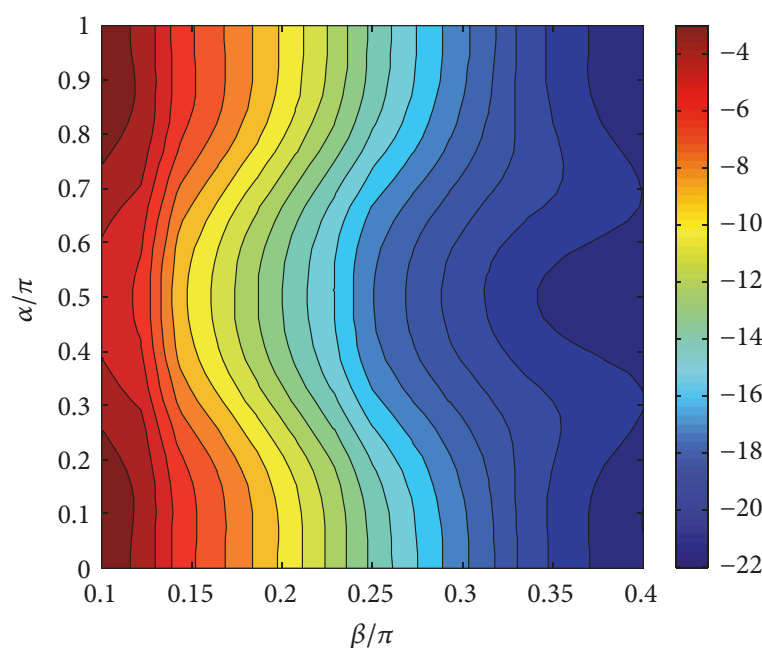

(a) $k_{s}^{m} a=2.07$

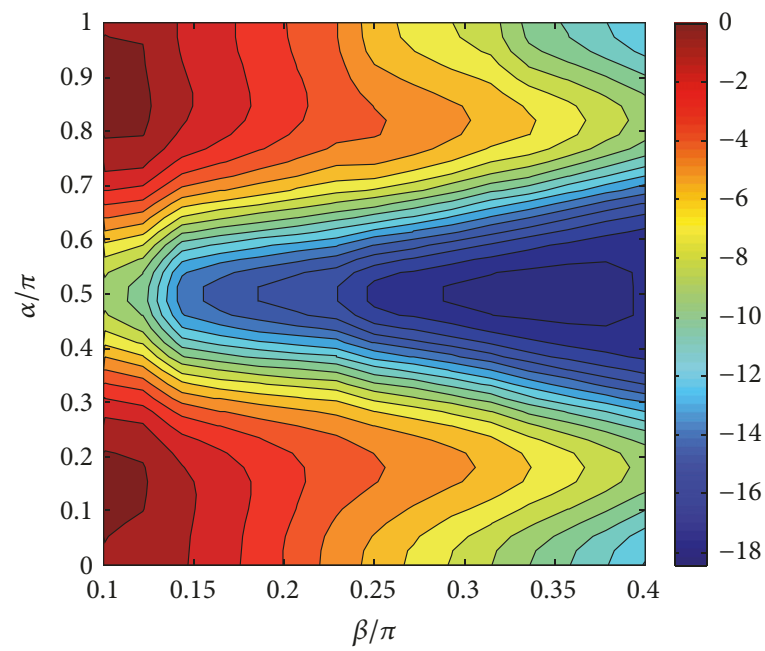

(c) $k_{s}^{m} a=4.15$

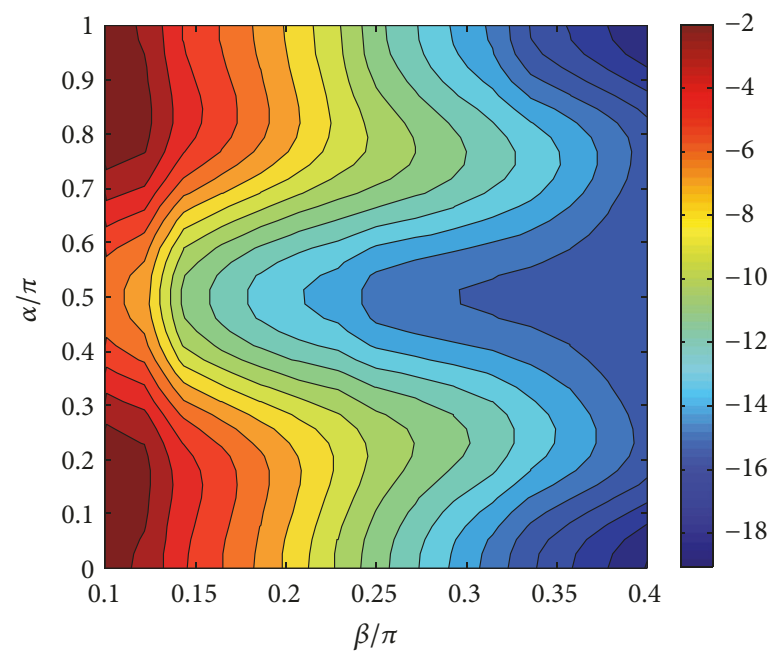

(b) $k_{s}^{m} a=3.11$

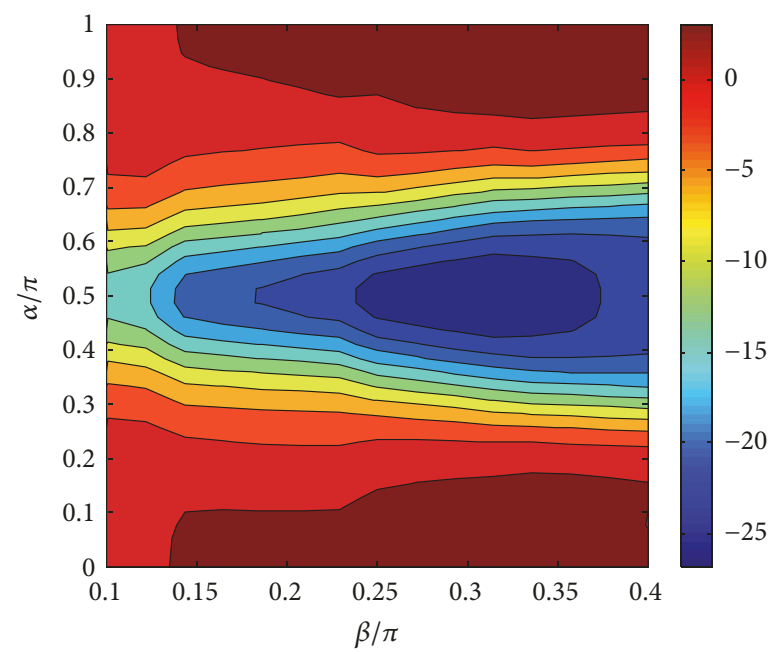

(d) $k_{l}^{m} a=5.18$

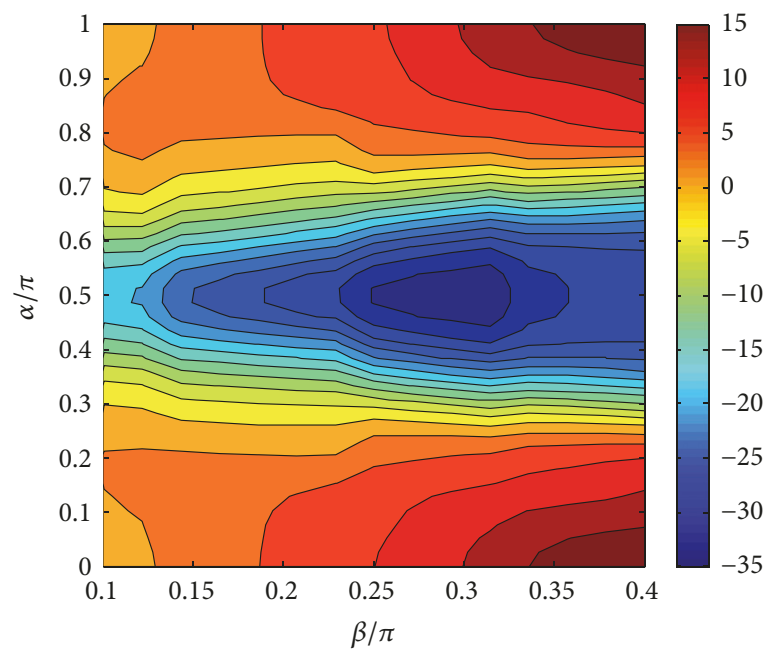

(e) $k_{l}^{m} a=6.22$

FIGURE 8: Relative difference (\%) of the calculated $\sigma_{s}$ by a fiber with a partially imperfect bonding and with the fully perfect bonding, under the SV-wave incidence at several different normalized frequencies. 


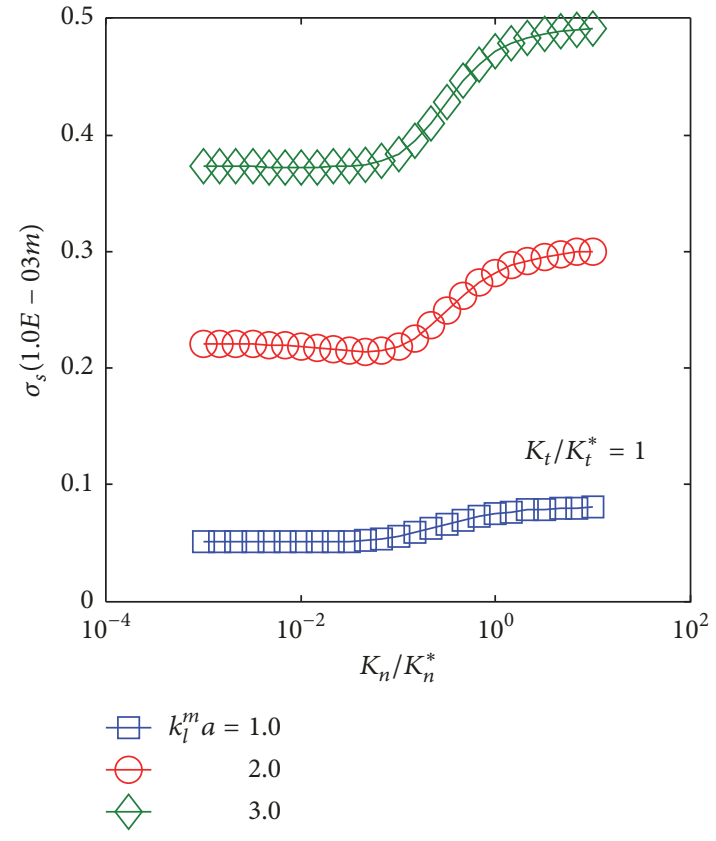

(a)

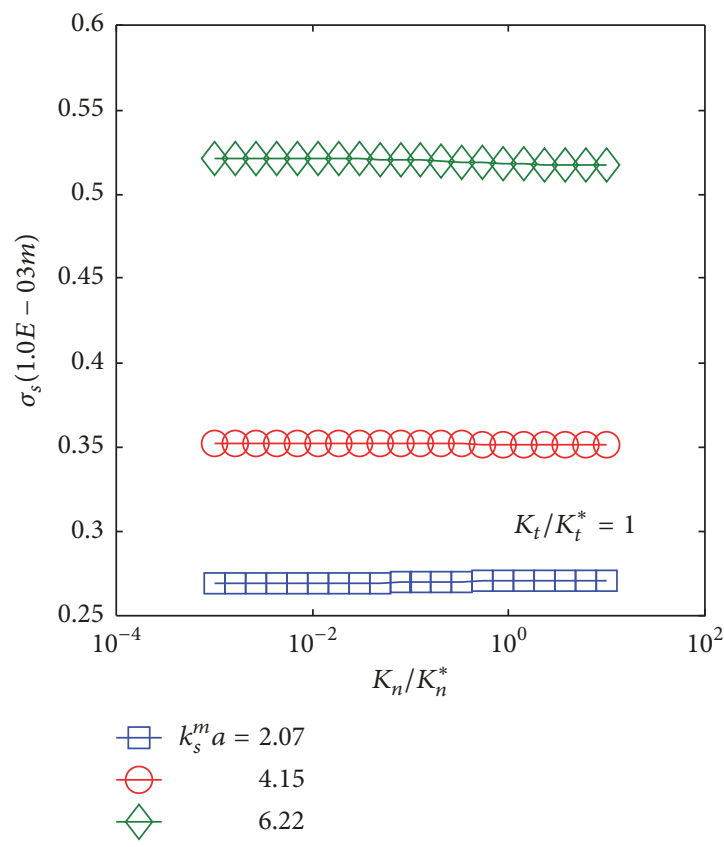

(c)

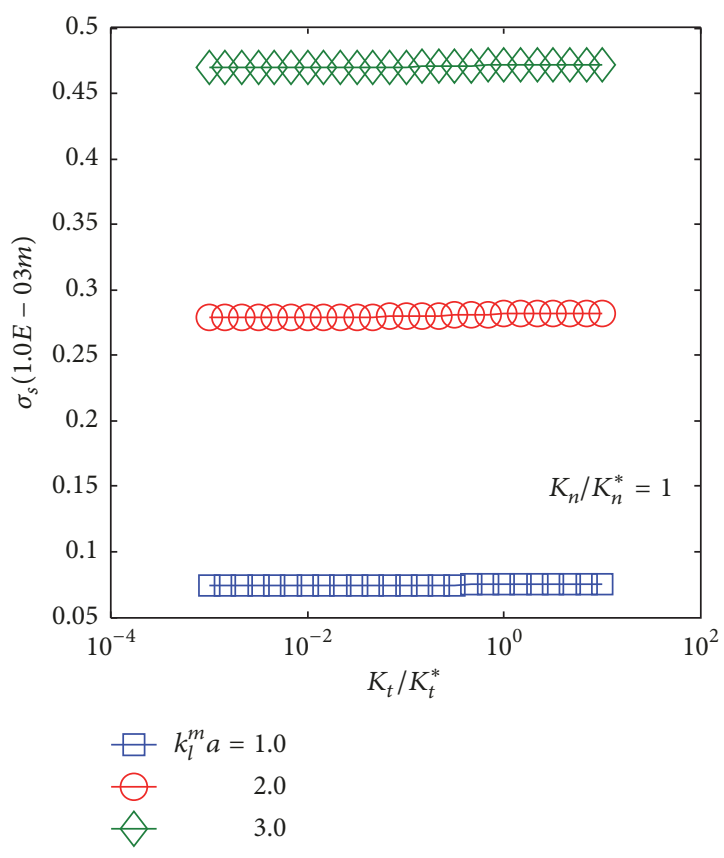

(b)

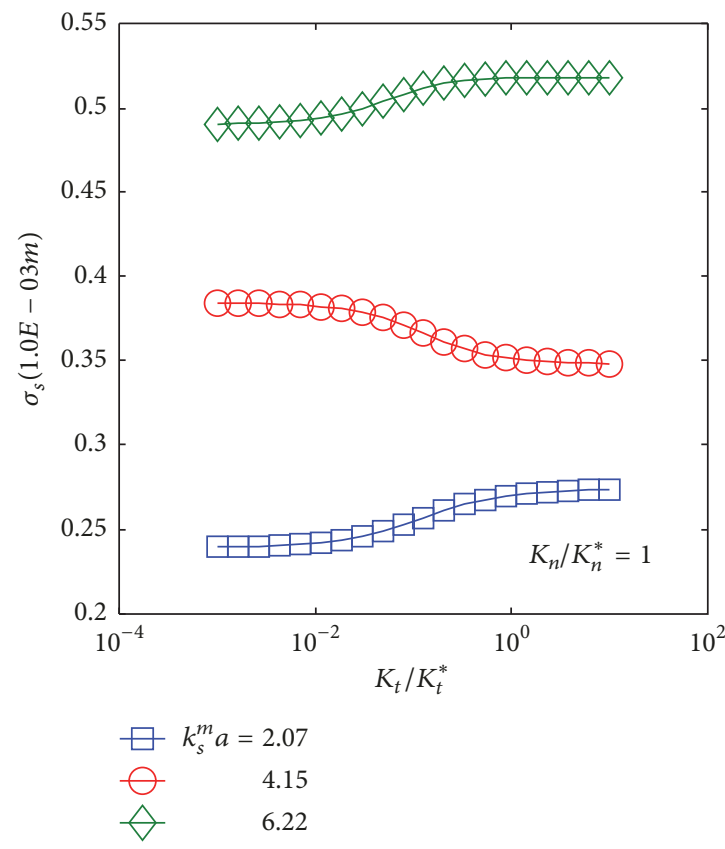

(d)

FIGURE 9: The influence of stiffness $K_{n}$ and stiffness $K_{t}$ on $\sigma_{s}$ under a P- and SV-wave incidence, respectively. (a) and (b) show results under the P-wave incidence and (c) and (d) show results under the SV-wave incidence. In (a) and (c) $K_{t} / K_{t}^{*}=1$, and in (b) and (d) $K_{n} / K_{n}^{*}=1$.

The corresponding results under the SV-wave incidence are plotted in Figure 12. Results show that $\sigma_{s}$ is sensitive to $K_{n}$ as $\alpha$ is larger, as Figure 12(a) shows. In contrast, $\sigma_{s}$ becomes sensitive to $K_{t}$ for smaller $\alpha$, as shown in Figure 12(b). Figure 12(c) shows the sensitivity of $\sigma_{s}$ to both $K_{n}$ and $K_{t}$ under the SV-wave incidence. Comparison of the results in Figures $11(c)$ and $12(c)$ indicates that when $\alpha$ is small, the P-wave incidence at higher frequencies is more applicable to evaluate the changing of the stiffness of the imperfectly bonded interface.
In contrast, the SV-wave incidence at higher frequencies is more applicable to evaluate the changing of the stiffness of the imperfectly bonded interface as $\alpha$ is large.

\section{Conclusions}

The single scattering of P- and SV-waves by a cylindrical fiber with a partially imperfect bonding to the matrix is investigated using the CP method. This study benefits the 


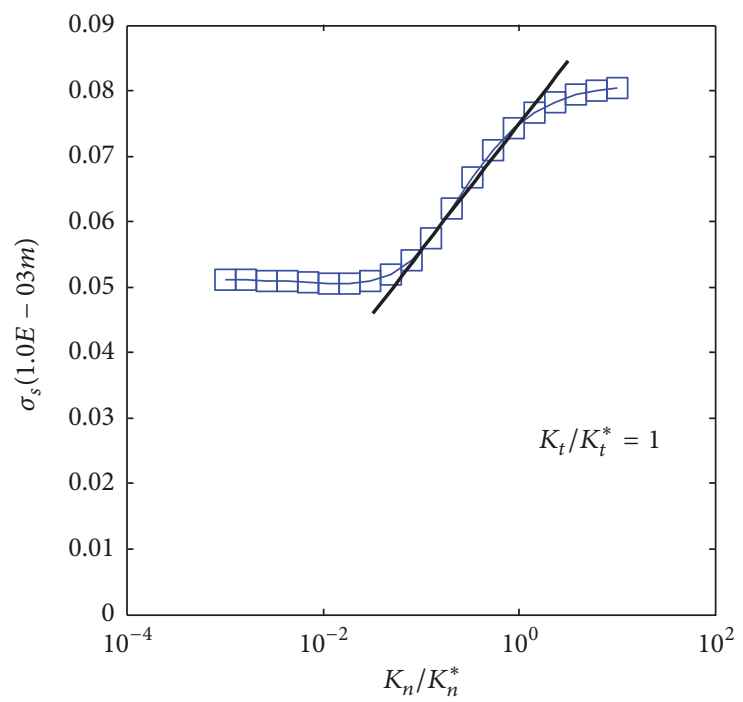

FigURE 10: A schematic to define the sensitivity of $\sigma_{s}$ to $K_{n}$ or $K_{t}$.

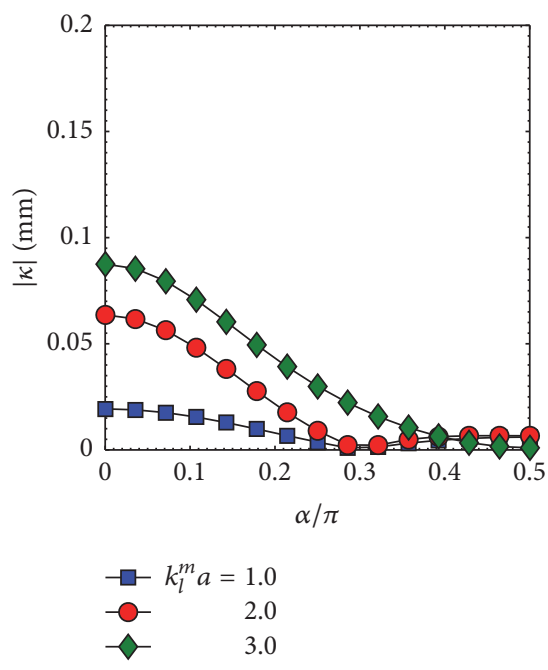

(a)

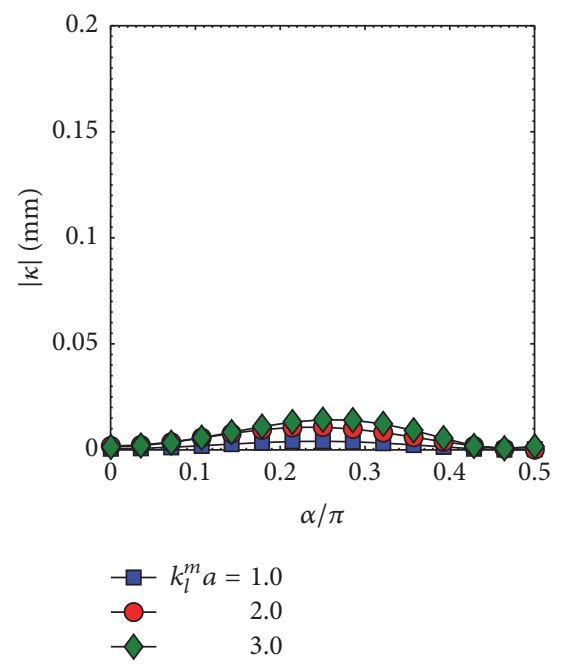

(b)

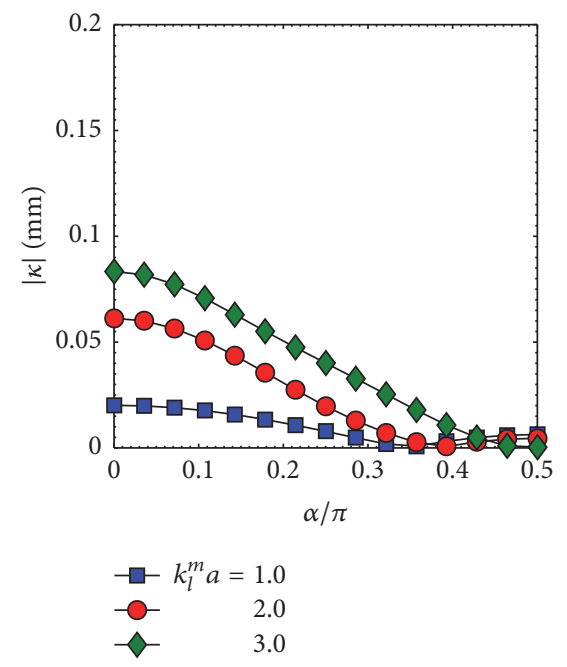

(c)

Figure 11: The sensitivity of $\sigma_{s}$ to (a) $K_{n}$ and (b) $K_{t}$ and (c) both $K_{n}$ and $K_{t}$, under the P-wave incidence. $K_{t} / K_{t}^{*}=1$ in (a), $K_{n} / K_{n}^{*}=1$ in (b), and $K_{n} / K_{n}^{*}=K_{t} / K_{t}^{*}$ in (c).

characterization of coherent waves in composites with randomly distributed inclusions. In this study, the imperfect interface is modelled using the spring model. Three examples are calculated and the results show that this CP method performs well. Also, the parametric analysis of location, width, and bonding level of the imperfect bonding on the total SCS is extensively studied. From this study, the following conclusions are obtained:

(a) The influence of the partially imperfect bonding on the single scattering of P- and SV-waves is almost symmetric about the axis $\alpha=\pi / 2$.

(b) Under the P-wave incidence, the $\sigma_{s}$ value reduced by the partially imperfect bonding at the location of $\alpha=0$ and $\pi$ is greater than that at any other location, compared with the fully perfect bonding. The partially imperfect bonding located at $\alpha=\pi / 2$ has almost no effect on $\sigma_{s}$. Under the SV-wave incidence, the partially imperfect bonding located at $\alpha=\pi / 2$ significantly reduces $\sigma_{s}$.

(c) When $\alpha$ is small, $\sigma_{s}$ under the $\mathrm{P}$-wave incidence has a high sensitivity to the level of the imperfect bonding. However, $\sigma_{s}$ under the SV-wave incidence becomes more sensitive to the level of the imperfect bonding as $\alpha$ is large.

In summary, besides its significance in the understanding of the single scattering, the present study also benefits for the application of the theoretical models to evaluate phase velocity and attenuation coefficient of coherent elastic waves 


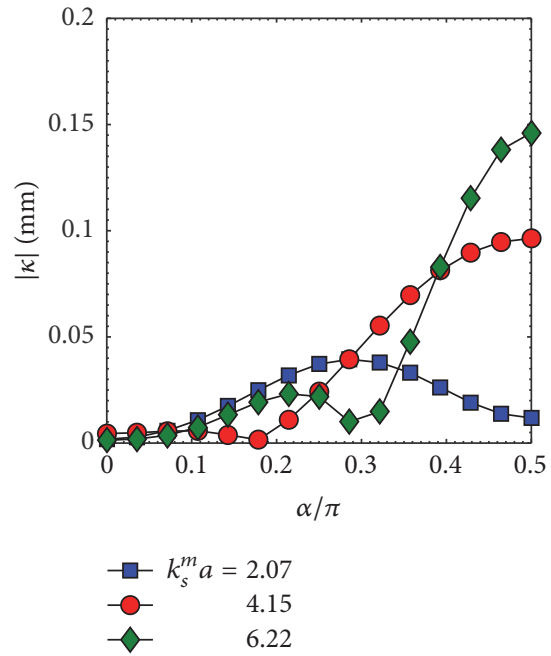

(a)

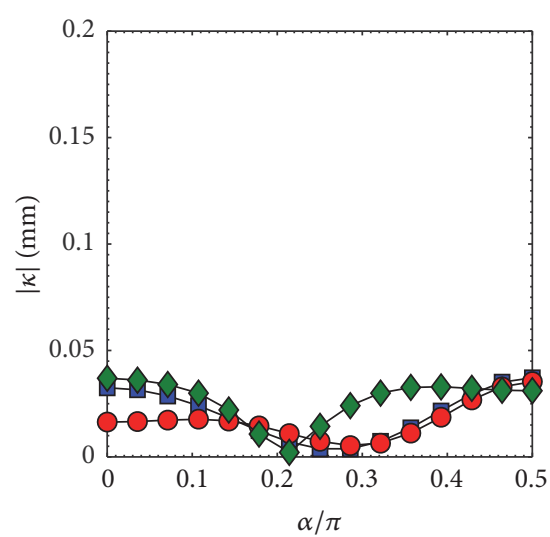

$\begin{array}{lr}\square & k_{s}^{m} a=2.07 \\ \neg & 4.15 \\ - & 6.22\end{array}$

(b)

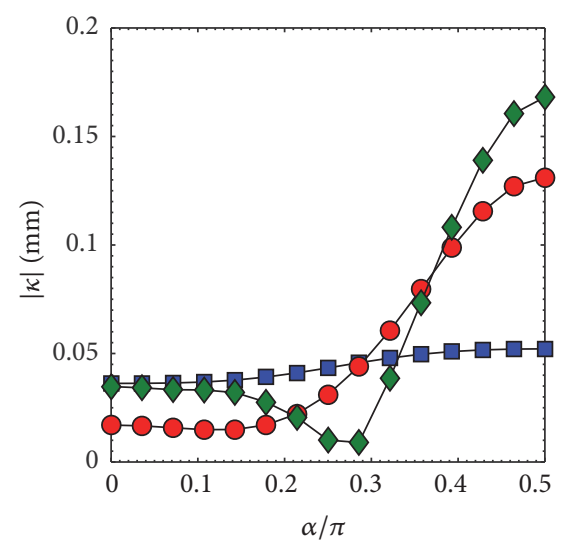

$\begin{array}{lr}\square & k_{s}^{m} a=2.07 \\ - & 4.15 \\ - & 6.22\end{array}$

(c)

FIGURE 12: The sensitivity of $\sigma_{s}$ to (a) $K_{n}$ and (b) $K_{t}$ and (c) both $K_{n}$ and $K_{t}$, under the SV-wave incidence. $K_{t} / K_{t}^{*}=1$ in (a), $K_{n} / K_{n}^{*}=1$ in (b), and $K_{n} / K_{n}^{*}=K_{t} / K_{t}^{*}$ in (c).

in composites with randomly distributed inclusions and the nondestructive evaluation of composites using the ultrasonic techniques.

\section{Appendix}

A. Expressions for $M_{i j}$ and $b_{i}$ in (7)

$$
\begin{aligned}
M_{31} & =\frac{\lambda^{m}+2 \mu^{m}}{\lambda^{m}} q_{l m}^{2} \cdot H_{n}^{(1)^{\prime \prime}}\left(q_{l m}\right)+(\mathbf{i} n)^{2} H_{n}^{(1)}\left(q_{l m}\right) \\
& +q_{l m} \cdot H_{n}^{(1)^{\prime}}\left(q_{l m}\right) \\
M_{32} & =\frac{2 \mu^{m}}{\lambda^{m}} \mathbf{i} n \cdot\left[q_{s m} \cdot H_{n}^{(1)^{\prime}}\left(q_{s m}\right)-H_{n}^{(1)}\left(q_{s m}\right)\right] \\
M_{33} & =-\left[\frac{\lambda^{f}+2 \mu^{f}}{\lambda^{m}} q_{l f}^{2} \cdot J_{n}^{\prime \prime}\left(q_{l f}\right)+\frac{\lambda^{f}}{\lambda^{m}}(\mathbf{i} n)^{2} J_{n}\left(q_{l f}\right)\right. \\
& \left.+\frac{\lambda^{f}}{\lambda^{m}} q_{l f} \cdot J_{n}^{\prime}\left(q_{l f}\right)\right] \\
M_{34} & =-\frac{2 \mu^{f}}{\lambda^{m}} \cdot \mathbf{i} n \cdot\left[q_{s f} \cdot J_{n}^{\prime}\left(q_{s f}\right)-J_{n}\left(q_{s f}\right)\right] \\
b_{3}= & -\mathbf{i}^{n}\left[\frac{\lambda^{m}+2 \mu^{m}}{\lambda^{m}} q_{l m}^{2} \cdot J_{n}^{\prime \prime}\left(q_{l m}\right)+(\mathbf{i} n)^{2} \cdot J_{n}\left(q_{l m}\right)\right. \\
& \left.+q_{l m} \cdot J_{n}^{\prime}\left(q_{l m}\right)\right] \\
M_{41} & =2 \mathbf{i} n \frac{\mu^{m}}{\mu^{f}}\left[-H_{n}^{(1)}\left(q_{l m}\right)+q_{l m} \cdot H_{n}^{(1)^{\prime}}\left(q_{l m}\right)\right]
\end{aligned}
$$

$$
\begin{aligned}
& M_{42}=\frac{\mu^{m}}{\mu^{f}}\left[(\mathbf{i} n)^{2} \cdot H_{n}^{(1)}\left(q_{s m}\right)-q_{s m}^{2} \cdot H_{n}^{(1)^{\prime \prime}}\left(q_{s m}\right)\right. \\
& \left.\quad+q_{s m} \cdot H_{n}^{(1)^{\prime}}\left(q_{s m}\right)\right] \\
& M_{43}=2 \mathbf{i} n\left[J_{n}\left(q_{l f}\right)-q_{l f} \cdot J_{n}^{\prime}\left(q_{l f}\right)\right] \\
& M_{44}=-\left[(\mathbf{i} n)^{2} \cdot J_{n}\left(q_{s f}\right)-q_{s f}^{2} \cdot J_{n}^{\prime \prime}\left(q_{s f}\right)+q_{s f}\right. \\
& \left.\cdot J_{n}^{\prime}\left(q_{s f}\right)\right] \\
& b_{4}=2 \mathbf{i}^{n} \cdot \mathbf{i} n \frac{\mu^{m}}{\mu^{f}}\left[J_{n}\left(q_{l m}\right)-q_{l m} \cdot J_{n}^{\prime}\left(q_{l m}\right)\right]
\end{aligned}
$$

with $q_{l m}=k_{l}^{m} a, q_{s m}=k_{s}^{m} a, q_{l f}=k_{l}^{f} a$, and $q_{s f}=k_{s}^{f} a$.

\section{B. Expressions for $c_{i j}$ and $h_{i}$ in (9)}

$$
\begin{aligned}
c_{11} & =\left\{\frac{\left(\lambda^{f}+2 \mu^{f}\right)}{\lambda^{f}} q_{l f}^{2} \cdot J_{n}^{\prime \prime}\left(q_{l f}\right)+(\mathbf{i} n)^{2} J_{n}\left(q_{l f}\right)+q_{l f}\right. \\
\cdot & J_{n}^{\prime}\left(q_{l f}\right)-\frac{K_{n} a}{\lambda^{f}}\left[\alpha_{11} H_{n}^{(1)^{\prime}}\left(q_{l m}\right) \cdot q_{l m}\right. \\
& \left.\left.+\alpha_{21} H_{n}^{(1)}\left(q_{s m}\right) \mathbf{i} n-q_{l f} \cdot J_{n}^{\prime}\left(q_{l f}\right)\right]\right\} e^{\mathrm{i} n \theta} \\
c_{12} & =\left\{\frac{2 \mu^{f}}{\lambda^{f}}\left[\mathbf{i} n \cdot q_{s f} \cdot J_{n}^{\prime}\left(q_{s f}\right)-\mathbf{i} n J_{n}\left(q_{s f}\right)\right]\right. \\
& -\frac{K_{n} a}{\lambda^{f}}\left[\alpha_{12} H_{n}^{(1)^{\prime}}\left(q_{l m}\right) \cdot q_{l m}+\alpha_{22} H_{n}^{(1)}\left(q_{s m}\right) \mathbf{i} n\right.
\end{aligned}
$$




$$
\begin{aligned}
& \left.\left.-\mathbf{i} n J_{n}\left(q_{s f}\right)\right]\right\} e^{\mathbf{i} n \theta} \\
& h_{1}=\frac{K_{n} a}{\lambda^{f}}\left\{\left[\mathbf{i}^{n} J_{n}^{\prime}\left(q_{l m}\right)+\beta_{1} H_{n}^{(1)^{\prime}}\left(q_{l m}\right)\right] q_{l m}\right. \\
& \left.+\beta_{2} H_{n}^{(1)}\left(q_{s m}\right) \mathbf{i} n\right\} e^{\mathrm{i} n \theta} \\
& c_{21}=\left[\alpha_{11} H_{n}^{(1)^{\prime}}\left(q_{l m}\right) \cdot q_{l m}+\alpha_{21} H_{n}^{(1)}\left(q_{s m}\right) \mathbf{i} n-q_{l f}\right. \\
& \left.\cdot J_{n}^{\prime}\left(q_{l f}\right)\right] e^{\mathrm{i} n \theta} \\
& c_{22}=\left[\alpha_{12} H_{n}^{(1)^{\prime}}\left(q_{l m}\right) \cdot q_{l m}+\alpha_{22} H_{n}^{(1)}\left(q_{s m}\right) \mathbf{i} n\right. \\
& \left.-\mathbf{i} n J_{n}\left(q_{s f}\right)\right] e^{\mathbf{i} n \theta} \\
& h_{2}=-\left[\dot{i}^{n} J_{n}^{\prime}\left(q_{l m}\right) \cdot q_{l m}+\beta_{1} H_{n}^{(1)^{\prime}}\left(q_{l m}\right) q_{l m}\right. \\
& \left.+\beta_{2} H_{n}^{(1)}\left(q_{s m}\right) \mathbf{i} n\right] e^{\mathrm{i} n \theta} \\
& c_{31}=\left\{\frac{\mu^{f}}{K_{t} a}\left[-2 \mathbf{i} n J_{n}\left(q_{l f}\right)+2 \mathbf{i} n q_{l f} J_{n}^{\prime}\left(q_{l f}\right)\right]\right. \\
& -\alpha_{11} H_{n}^{(1)}\left(q_{l m}\right) \mathrm{i} n+\alpha_{21} q_{s m} \cdot H_{n}^{(1)^{\prime}}\left(q_{s m}\right) \\
& \left.+\mathbf{i} n J_{n}\left(q_{l f}\right)\right\} e^{\mathrm{i} n \theta} \\
& c_{32}=\left\{\frac { \mu ^ { f } } { K _ { t } a } \left[(\mathbf{i} n)^{2} J_{n}\left(q_{s f}\right)-q_{s f}^{2} \cdot J_{n}^{\prime \prime}\left(q_{s f}\right)+q_{s f}\right.\right. \\
& \left.\cdot J_{n}^{\prime}\left(q_{s f}\right)\right]-\alpha_{12} H_{n}^{(1)}\left(q_{l m}\right) \mathrm{i} n+\alpha_{22} q_{s m} H_{n}^{(1)^{\prime}}\left(q_{s m}\right) \\
& \left.-q_{s f} \cdot J_{n}^{\prime}\left(q_{s f}\right)\right\} e^{\mathrm{i} n \theta} \\
& h_{3}=\left\{\left[\mathbf{i}^{n} J_{n}\left(q_{l m}\right)+\beta_{1} H_{n}^{(1)}\left(q_{l m}\right)\right] \mathbf{i} n-\beta_{2} q_{s m}\right. \\
& \left.\cdot H_{n}^{(1)^{\prime}}\left(q_{s m}\right)\right\} e^{\mathrm{i} n \theta} \\
& c_{41}=\left[\alpha_{11} H_{n}^{(1)}\left(q_{l m}\right) \mathbf{i} n-\alpha_{21} q_{s m} \cdot H_{n}^{(1)^{\prime}}\left(q_{s m}\right)\right. \\
& \left.-\mathbf{i} n J_{n}\left(q_{l f}\right)\right] e^{\mathrm{i} n \theta} \\
& c_{42}=\left[\alpha_{12} H_{n}^{(1)}\left(q_{l m}\right) \text { i } n-\alpha_{22} q_{s m} \cdot H_{n}^{(1)^{\prime}}\left(q_{s m}\right)+q_{s f}\right. \\
& \left.\cdot J_{n}^{\prime}\left(q_{s f}\right)\right] e^{\mathrm{i} n \theta} \\
& h_{4}=-\left\{\left[\dot{i}^{n} J_{n}\left(q_{l m}\right)+\beta_{1} H_{n}^{(1)}\left(q_{l m}\right)\right] \mathbf{i} n-\beta_{2} q_{s m}\right. \\
& \left.\cdot H_{n}^{(1)^{\prime}}\left(q_{s m}\right)\right\} e^{\mathrm{i} n \theta} .
\end{aligned}
$$

It is noted that Appendices A and B only list the expressions of the parameters under the P-wave incidence. Expressions for the SV-incidence can be obtained without essential difficulties.

\section{Data Availability}

The data used to support the findings of this study are available from the corresponding author upon request.

\section{Conflicts of Interest}

The authors declare that they have no conflicts of interest.

\section{Acknowledgments}

This work was supported by the National Natural Science Foundation of China (Grants 11502036 and 51708434) and the Natural Science Fund of the City of Chongqing (Grant cstc2015jcyjA0577). Guangxi Key Laboratory of Manufacturing Systems and Advanced Manufacturing Technology (no. 16-380-12-014k) also provided partial financial support.

\section{References}

[1] J. Zhang, N. Hu, and J. Zhang, "Independent scattering model for evaluating antiplane shear wave attenuation in fiber-reinforced composite materials," Ultrasonics, vol. 78, pp. 185-194, 2017.

[2] J. Zhang and W. Ye, "An explicit formula for the coherent SH waves' attenuation coefficient in random porous materials with low porosities," Ultrasonics, vol. 62, pp. 27-34, 2015.

[3] T. Sumiya, S. Biwa, and G. Haiat, "Computational multiple scattering analysis of elastic waves in unidirectional composites, Wave Motion," An International Journal Reporting Research on Wave Phenomena, vol. 50, no. 2, pp. 253-270, 2013.

[4] S. Biwa, S. Yamamoto, F. Kobayashi, and N. Ohno, "Computational multiple scattering analysis for shear wave propagation in unidirectional composites," International Journal of Solids and Structures, vol. 41, no. 2, pp. 435-457, 2004.

[5] S. I. Rokhlin, W. Huang, and Y. C. Chu, "Ultrasonic scattering and velocity methods for characterization of fibre-matrix interphases," Ultrasonics, vol. 33, no. 5, pp. 351-364, 1995.

[6] L. L. Shaw, P. Karpur, and T. E. Matikas, "Fracture strength and damage progression of the fiber/matrix interfaces in titaniumbased MMCs with different interfacial layers," Composites Part B: Engineering, vol. 29, no. 3, pp. 331-339, 1998.

[7] J. Aboudi, "Damage in composites-Modeling of imperfect bonding," Composites Science and Technology, vol. 28, no. 2, pp. 103128, 1987.

[8] S. I. Rokhlin, Y. C. Chu, and W. Huang, "Ultrasonic evaluation of fatigue damage in metal matrix composites," Mechanics of Materials, vol. 21, no. 4, pp. 251-263, 1995.

[9] J.-Y. Kim, "Attenuation and speed of antiplane shear wave in fiber-reinforced composites with random interfacial cracks," International Journal of Solids and Structures, vol. 38, no. 40-41, pp. 7121-7137, 2001.

[10] W. Liu and R. D. Kriz, "Shear waves in fiber-reinforced composites with interfacial cracks," International Journal of Solids and Structures, vol. 35, no. 13, pp. 1425-1449, 1998. 
[11] A. Norris and Y. Yang, "Dynamic stress on a partially bonded fiber," Journal of Applied Mechanics, vol. 58, no. 2, pp. 404-409, 1991.

[12] Y. Yang and A. N. Norris, "Shear wave scattering from a debonded fibre," Journal of the Mechanics and Physics of Solids, vol. 39, no. 2, pp. 273-294, 1991.

[13] Y. Yang and A. Norris, "Longitudinal wave scattering from a partially bonded fiber," Wave Motion, vol. 15, no. 1, pp. 43-59, 1992.

[14] Y. Benveniste and J. Aboudi, "A continuum model for fiber reinforced materials with debonding," International Journal of Solids and Structures, vol. 20, no. 11-12, pp. 935-951, 1984.

[15] X.-Q. Fang, H.-X. Jin, and B.-L. Wang, "Dynamic interaction of two circular lined tunnels with imperfect interfaces under cylindrical P-waves," International Journal of Rock Mechanics and Mining Sciences, vol. 79, pp. 172-182, 2015.

[16] X.-Q. Fang, H.-C. Huang, and J.-X. Liu, "Predicting the crack opening displacements of a partially debonded pipeline in rock mass under P waves," Fatigue \& Fracture of Engineering Materials \& Structures, vol. 39, no. 7, pp. 886-895, 2016.

[17] Y. S. Wang and D. Wang, "Scattering of elastic waves by a rigid cylindrical inclusion partially debonded from its surrounding matrix - I. SH case," International Journal of Solids and Structures, vol. 33, no. 19, pp. 2789-2815, 1996.

[18] Y. S. Wang and D. Wang, "Scattering of elastic waves by a rigid cylindrical inclusion partially debonded from its surrounding matrix - II. P and SV cases," International Journal of Solids and Structures, vol. 33, no. 19, pp. 2817-2840, 1996.

[19] S. Biwa and T. Shibata, "Elastic and ultrasonic properties of a unidirectional composite with partially debonded fibres: Numerical analysis for longitudinal shear modes," Composites Science and Technology, vol. 60, no. 1, pp. 83-93, 2000.

[20] S. Biwa and T. Shibata, "Influence of interface debonding on ultrasonic phase velocity and atténuation in a fiber-reinforced composite - scattering analysis for shear waves polarized parallel with fibers -," Zairyo/Journal of the Society of Materials Science, Japan, vol. 47, no. 4, pp. 399-405, 1998.

[21] H. Sato and Y. Shindo, "Influence of microstructure on scattering of plane elastic waves by a distribution of partially debonded elliptical inclusions," Mechanics of Materials, vol. 34, no. 7, pp. 401-409, 2002.

[22] N. M. Newmark, "Tests and analysis of composite beams with incomplete interaction," in Proceedings of the Society for Experimental Stress Analysis, vol. 9, pp. 75-92, 1951.

[23] P. A. Martin, "Boundary integral equations for the scattering of elastic waves by elastic inclusions with thin interface layers," Journal of Nondestructive Evaluation, vol. 11, no. 3-4, pp. 167-174, 1992.

[24] X.-Q. Fang and H.-X. Jin, "Dynamic response of a non-circular lined tunnel with visco-elastic imperfect interface in the saturated poroelastic medium," Computers \& Geosciences, vol. 83, pp. 98-105, 2017.

[25] H. Brito-Santana, R. de Medeiros, R. Rodriguez-Ramos, and V. Tita, "Different interface models for calculating the effective properties in piezoelectric composite materials with imperfect fiber-matrix adhesion," Composite Structures, vol. 151, pp. 7080, 2016.

[26] A. K. Mal and S. K. Bose, "Dynamic elastic moduli of a suspension of imperfectly bonded spheres," Mathematical Proceedings of the Cambridge Philosophical Society, vol. 76, no. 3, pp. 587600, 1974 .
[27] E. Vilchevskaya and I. Sevostianov, "Overall thermal conductivity of a fiber reinforced composite with partially debonded inhomogeneities," International Journal of Engineering Science, vol. 98, pp. 99-109, 2016.

[28] W. Huang, S. Brisuda, and S. I. Rokhlin, "Ultrasonic wave scattering from fiber-matrix interphases," The Journal of the Acoustical Society of America, vol. 97, no. 2, pp. 807-817, 1995.

[29] X.-Q. Fang, H.-X. Jin, J.-X. Liu, and M.-J. Huang, "Imperfect bonding effect on dynamic response of a non-circular lined tunnel subjected to shear waves," Tunnelling and Underground Space Technology, vol. 56, pp. 226-231, 2016.

[30] X.-Q. Fang and H.-X. Jin, "Visco-elastic imperfect bonding effect on dynamic response of a non-circular lined tunnel subjected to P and SV waves," Soil Dynamics and Earthquake Engineering, vol. 88, pp. 1-7, 2016.

[31] J. C. Lopez-Realpozo, R. Rodriguez-Ramos, R. Guinovart-Diaz et al., "Effective elastic shear stiffness of a periodic fibrous composite with non-uniform imperfect contact between the matrix and the fibers," International Journal of Solids and Structures, vol. 51, no. 6, pp. 1253-1262, 2014.

[32] R. Guinovart-Díaz, R. Rodríguez-Ramos, J. C. López-Realpozo et al., "Analysis of fibrous elastic composites with nonuniform imperfect adhesion," Acta Mechanica, vol. 227, no. 1, pp. 57-73, 2016.

[33] C. C. Mow and Y. H. Pao, The Diffraction of Elastic Waves and Dynamic Stress Concentrations, vol. 40, 1971.

[34] P. C. Waterman and R. Truell, "Multiple scattering of waves," Journal of Mathematical Physics, vol. 2, pp. 512-537, 1961.

[35] V. K. Varadan, V. V. Varadan, and Y. . Pao, "Multiple scattering of elastic waves by cylinders of arbitrary cross section part I. SH waves," The Journal of the Acoustical Society of America, vol. 63, no. 5, pp. 1310-1319, 1978.

[36] V. K. Varadan, Y. Ma, and V. V. Varadan, "A multiple scattering theory for elastic wave propagation in discrete random media," The Journal of the Acoustical Society of America, vol. 77, no. 2, pp. 375-385, 1985.

[37] S. K. Kanaun and V. M. Levin, "Self-consistent methods in the problem of axial elastic shear wave propagation through fiber composites," Archive of Applied Mechanics, vol. 73, no. 1-2, pp. 105-130, 2003.

[38] J.-Y. Kim, J.-G. Ih, and B.-H. Lee, "Dispersion of elastic waves in random particulate composites," The Journal of the Acoustical Society of America, vol. 97, no. 3, pp. 1380-1388, 1995.

[39] X.-Q. Fang, C. Hu, and W.-H. Huang, "Dynamic effective properties of semi-infinite random unidirectional fiber-reinforced composites subjected to anti-plane shear waves," Composite Structures, vol. 83, no. 2, pp. 159-167, 2008.

[40] S. K. Kanaun and V. M. Levin, "Effective medium method in the problem of axial elastic shear wave propagation through fiber composites," International Journal of Solids and Structures, vol. 40, no. 18, pp. 4859-4878, 2003.

[41] S. Biwa, "Independent scattering and wave attenuation in viscoelastic composites," Mechanics of Materials, vol. 33, no. 11, pp. 635-647, 2001.

[42] S. Biwa, S. Idekoba, and N. Ohno, "Wave attenuation in particulate polymer composites: Independent scattering/absorption analysis and comparison to measurements," Mechanics of Materials, vol. 34, no. 10, pp. 671-682, 2002. 


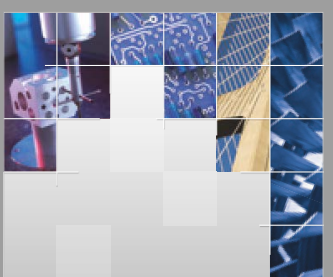

\section{Enfincering}
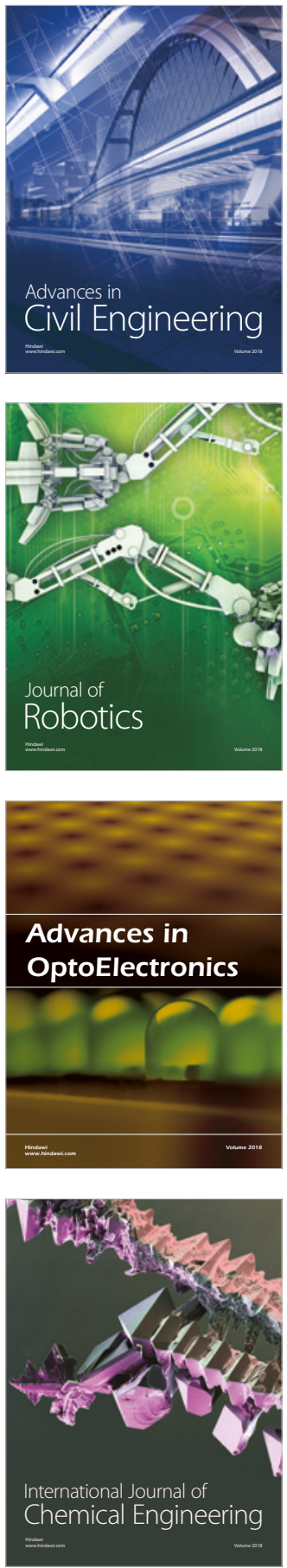

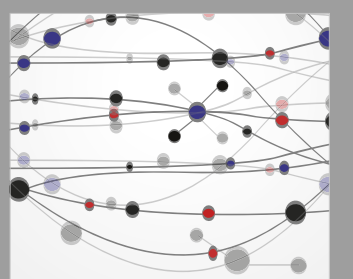

\section{Rotating \\ Machinery}

The Scientific World Journal

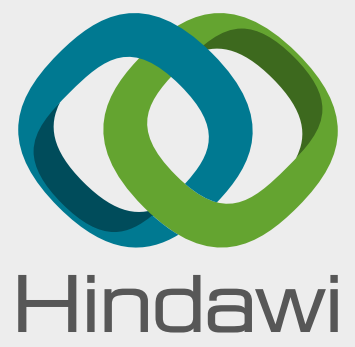

Submit your manuscripts at

www.hindawi.com
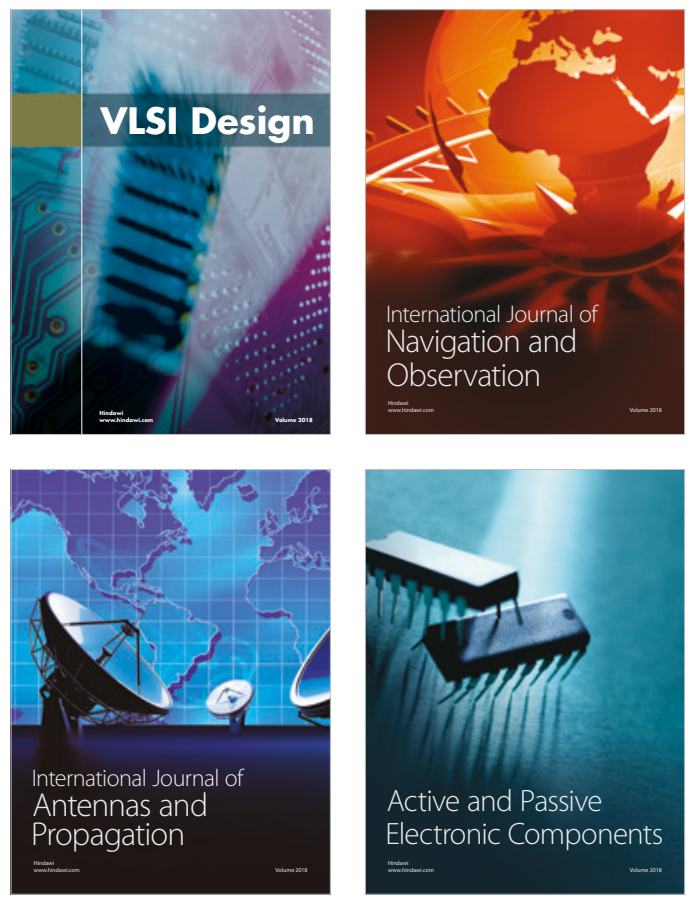
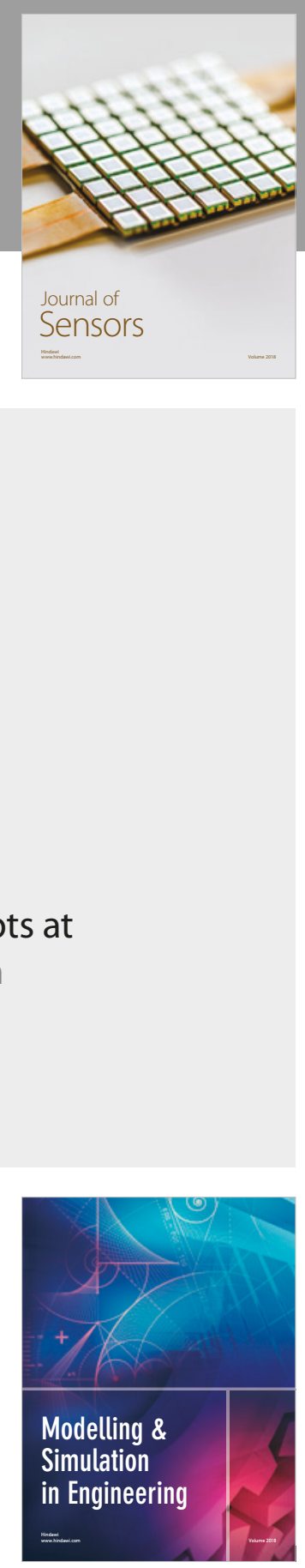

\section{Advances \\ Multimedia}
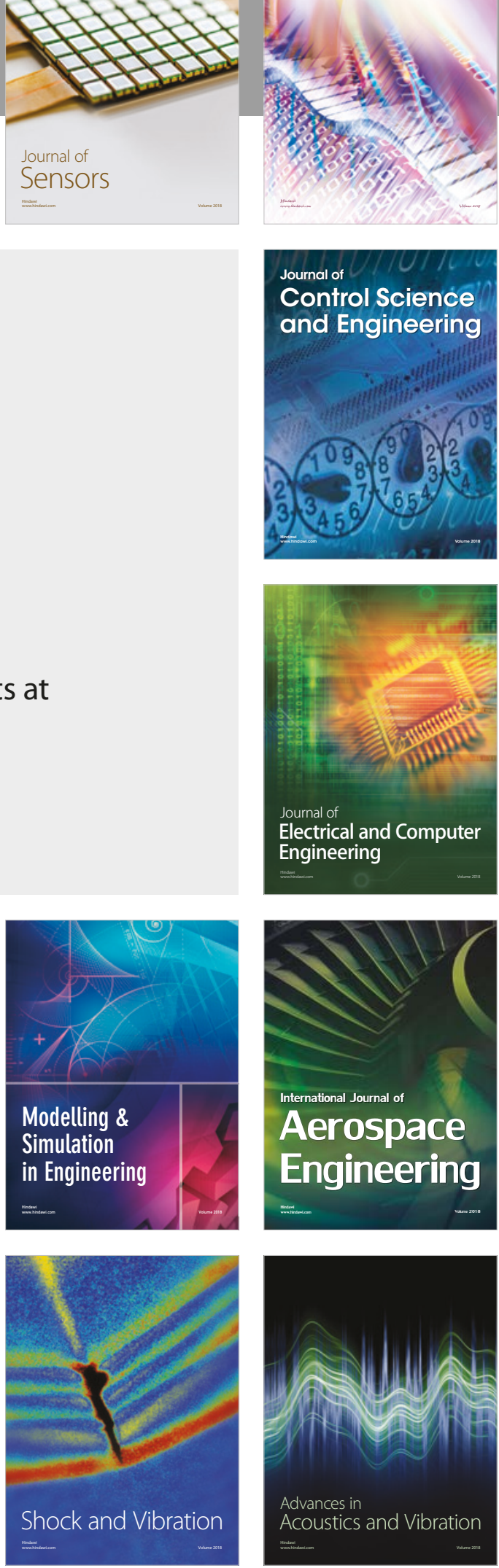